Universidad

:

BIBLIOTECA

Document downloaded from the institutional repository of the University of Alcala: https://ebuah.uah.es/dspace/

This is a postprint version of the following published document:

Martín, A. et al., 2006. Cube-type nitrido complexes containing titanium and zinc/copper. Inorganic chemistry, 45(17), pp.6901-6911.

Available at https://doi.org/10.1021/ic0603407

(c) 2006 American Chemical Society.
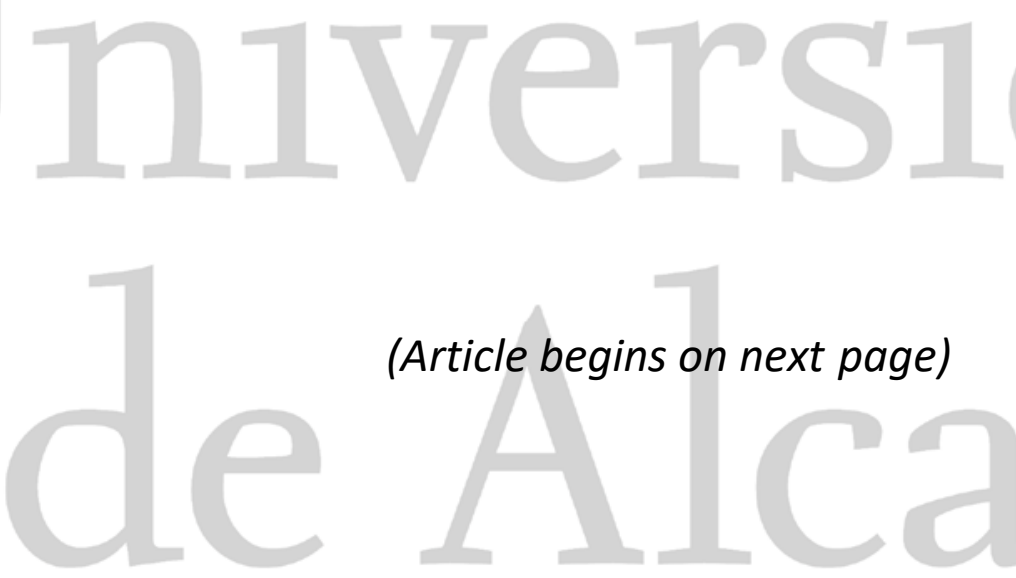

(Article begins on next page)

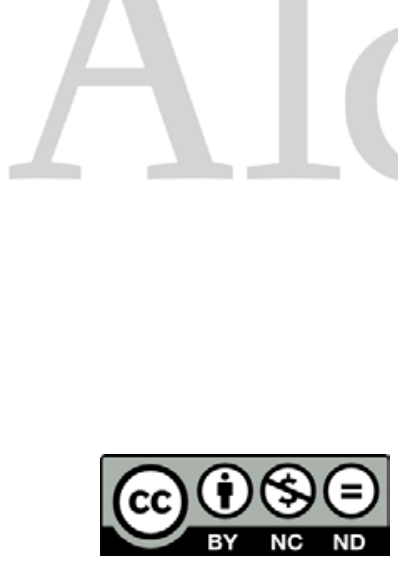

This work is licensed under a

Creative Commons Attribution-NonCommercial-NoDerivatives

4.0 International License. 


\section{Cube-Type Nitrido Complexes Containing Titanium and Zinc/Copper}

Avelino Martín, Noelia Martínez-Espada, Miguel Mena, Adrián Pérez-Redondo, and Carlos Yélamos*

Departamento de Química Inorgánica, Universidad de Alcalá. 28871 Alcalá de HenaresMadrid (Spain). FAX: (+34) 91-8854683. E-mail: carlos.yelamos@uah.es

\section{Synopsis:}

The imido-nitrido complex $\left[\left\{\mathrm{Ti}\left(\eta^{5}-\mathrm{C}_{5} \mathrm{Me}_{5}\right)(\mu-\mathrm{NH})\right\}_{3}\left(\mu_{3}-\mathrm{N}\right)\right](\mathbf{1})$ acts as a tridentate ligand towards zinc(II) or copper(I) $\left[\mathrm{MX}_{n}\right]$ derivatives in its neutral $\left\{\left(\mu_{3}-\mathrm{NH}\right)_{3} \mathrm{Ti}_{3}\left(\eta^{5}-\mathrm{C}_{5} \mathrm{Me}_{5}\right)_{3}\left(\mu_{3}-\mathrm{N}\right)\right\}$
(A) or monoanionic $\left\{\left(\mu_{3}-\mathrm{N}\right)\left(\mu_{3}-\mathrm{NH}\right)_{2} \mathrm{Ti}_{3}\left(\eta^{5}-\mathrm{C}_{5} \mathrm{Me}_{5}\right)_{3}\left(\mu_{3}-\mathrm{N}\right)\right\}^{-}$
(B) form. The new heterometallic nitrido compounds exhibit single cube structures, excepting the copper derivative $\left[\left\{\mathrm{Cu}\left(\mu_{4}-\mathrm{N}\right)\left(\mu_{3}-\mathrm{NH}\right)_{2} \mathrm{Ti}_{3}\left(\eta^{5}-\mathrm{C}_{5} \mathrm{Me}_{5}\right)_{3}\left(\mu_{3}-\mathrm{N}\right)\right\}_{2}\right]$ (C) that shows an edge-linked double-cube structure with a $\left[\mathrm{Cu}_{2} \mathrm{Ti}_{6} \mathrm{~N}_{8}\right]$ core.

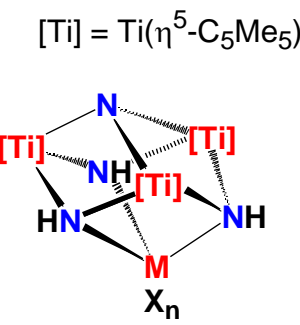

(A) $\quad \mathrm{M}=\mathrm{Zn}, \mathrm{Cu}$

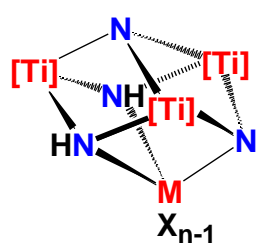

(B)

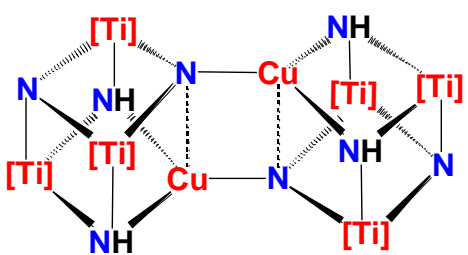

(C) 


\section{Abstract:}

Several azaheterometallocubane complexes containing $\left[\mathrm{MTi}_{3} \mathrm{~N}_{4}\right]$ cores have been prepared by the reaction of $\left[\left\{\mathrm{Ti}\left(\eta^{5}-\mathrm{C}_{5} \mathrm{Me}_{5}\right)(\mu-\mathrm{NH})\right\}_{3}\left(\mu_{3}-\mathrm{N}\right)\right]$ (1) with zinc(II) and copper(I) derivatives. Treatment of $\mathbf{1}$ with zinc dichloride in toluene at room temperature affords the adduct $\left[\mathrm{Cl}_{2} \mathrm{Zn}\left\{\left(\mu_{3}-\mathrm{NH}\right)_{3} \mathrm{Ti}_{3}\left(\eta^{5}-\mathrm{C}_{5} \mathrm{Me}_{5}\right)_{3}\left(\mu_{3}-\mathrm{N}\right)\right\}\right]$ (2). Attempts to crystallize $\mathbf{2}$ in dichloromethane gave yellow crystals of the ammonia adduct $\left[\left(\mathrm{H}_{3} \mathrm{~N}\right) \mathrm{Cl}_{2} \mathrm{Zn}\left\{\left(\mu_{3}-\mathrm{NH}\right) \mathrm{Ti}_{3}\left(\eta^{5}-\mathrm{C}_{5} \mathrm{Me}_{5}\right)_{3}(\mu-\mathrm{NH})_{2}\left(\mu_{3}-\right.\right.\right.$ N)\}] (3). The analogous reaction of $\mathbf{1}$ with alkyl, (trimethylsilyl)cyclopentadienyl or amido zinc complexes $\left[\mathrm{ZnR}_{2}\right]$ leads to the cube-type derivatives $\left[\operatorname{RZn}\left\{\left(\mu_{3}-\mathrm{N}\right)\left(\mu_{3}-\mathrm{NH}\right)_{2} \mathrm{Ti}_{3}\left(\eta^{5}-\right.\right.\right.$ $\left.\left.\left.\mathrm{C}_{5} \mathrm{Me}_{5}\right)_{3}\left(\mu_{3}-\mathrm{N}\right)\right\}\right]\left(\mathrm{R}=\mathrm{CH}_{2} \mathrm{SiMe}_{3}(\mathbf{5}), \mathrm{CH}_{2} \mathrm{Ph}(\mathbf{6}), \mathrm{Me}(\mathbf{7}), \mathrm{C}_{5} \mathrm{H}_{4} \mathrm{SiMe}_{3}(\mathbf{8}), \mathrm{N}\left(\mathrm{SiMe}_{3}\right)_{2}(\mathbf{9})\right)$ via RH elimination. The amido complex 9 decomposes in presence of ambient light to generate the alkyl derivative $\left[\left\{\mathrm{Me}_{3} \mathrm{Si}(\mathrm{H}) \mathrm{N}(\mathrm{Me})_{2} \mathrm{SiCH}_{2}\right\} \mathrm{Zn}\left\{\left(\mu_{3}-\mathrm{N}\right)\left(\mu_{3}-\mathrm{NH}\right)_{2} \mathrm{Ti}_{3}\left(\eta^{5}-\mathrm{C}_{5} \mathrm{Me}_{5}\right)_{3}\left(\mu_{3}-\mathrm{N}\right)\right\}\right]$ (10). The chloride complex 2 reacts with lithium cyclopentadienyl or lithium indenyl reagents to give the cyclopentadienyl or indenyl zinc derivatives $\left[\operatorname{RZn}\left\{\left(\mu_{3}-\mathrm{N}\right)\left(\mu_{3}-\mathrm{NH}\right)_{2} \mathrm{Ti}_{3}\left(\eta^{5}-\right.\right.\right.$ $\left.\left.\left.\mathrm{C}_{5} \mathrm{Me}_{5}\right)_{3}\left(\mu_{3}-\mathrm{N}\right)\right\}\right]\left(\mathrm{R}=\mathrm{C}_{5} \mathrm{H}_{5}(\mathbf{1 1}), \mathrm{C}_{9} \mathrm{H}_{7}(\mathbf{1 2})\right)$. Treatment of 1 with copper(I) halides in toluene at room temperature leads to the adducts $\left[\mathrm{XCu}\left\{\left(\mu_{3}-\mathrm{NH}\right)_{3} \mathrm{Ti}_{3}\left(\eta^{5}-\mathrm{C}_{5} \mathrm{Me}_{5}\right)_{3}\left(\mu_{3}-\mathrm{N}\right)\right\}\right](\mathrm{X}=\mathrm{Cl}(\mathbf{1 3})$, I (14)). Complex 13 reacts with lithium bis(trimethylsilyl)amido in toluene to give the precipitation of $\left[\left\{\mathrm{Cu}\left(\mu_{4}-\mathrm{N}\right)\left(\mu_{3}-\mathrm{NH}\right)_{2} \mathrm{Ti}_{3}\left(\eta^{5}-\mathrm{C}_{5} \mathrm{Me}_{5}\right)_{3}\left(\mu_{3}-\mathrm{N}\right)\right\}_{2}\right](\mathbf{1 5})$. Complex 15 is prepared in higher yield through the reaction of $\mathbf{1}$ with $\left[\left\{\mathrm{CuN}\left(\mathrm{SiMe}_{3}\right)_{2}\right\}_{4}\right]$ in toluene at $150{ }^{\circ} \mathrm{C}$. Addition of triphenylphosphane to 15 in toluene affords the single cube compound $\left[\left(\mathrm{Ph}_{3} \mathrm{P}\right) \mathrm{Cu}\left\{\left(\mu_{3}-\mathrm{N}\right)\left(\mu_{3}-\right.\right.\right.$ $\left.\left.\mathrm{NH})_{2} \mathrm{Ti}_{3}\left(\eta^{5}-\mathrm{C}_{5} \mathrm{Me}_{5}\right)_{3}\left(\mu_{3}-\mathrm{N}\right)\right\}\right]$ (16). The X-ray crystal structures of $\mathbf{3}, \mathbf{8}, \mathbf{9}$ and $\mathbf{1 5}$ have been determined. 


\section{Introduction}

An extensive coordination chemistry has been developed with tridentate nitrogen-based ligands. Representative examples are tris(pyrazolyl)borates, ${ }^{1}$ tris(pyrazolyl)methanes, ${ }^{2}$ triazacyclononanes, ${ }^{3}$ triazacyclohexanes, ${ }^{4}$ and other tripodal amido ligands,${ }^{5}$ because of their ability to serve as facially coordinating six-electron donors. In this binding mode, the tris(pyrazolyl)hydroborate ligand is formally analogous to the cyclopentadienide $\left(\mathrm{Cp}^{-}\right)$ligand, in that both are uninegative and isolobal. However, the electron-donating ability ${ }^{6}$ and the steric profile of tris(pyrazolyl)hydroborate (Tp) ligand, and substituted $\left(\mathrm{Tp}^{\mathrm{x}}\right)$ derivatives thereof, are very different. ${ }^{7}$ At present, tris(pyrazolyl)hydroborates have become one of the most widely used class of ligand in inorganic chemistry, giving different reactivity patterns and frequently permitting the isolation of species whose cyclopentadienyl relatives are highly reactive. ${ }^{1}$ In comparison, the chemistry of tris(pyrazolyl)methane ligands, the neutral analogous of the anionic tris(pyrazolyl)hydroborates, has been underdeveloped, although this area is a subject of active research in nowadays. ${ }^{2}$ The neutral 1,4,7-triazacyclononane $\left(\mathrm{R}_{3} \operatorname{tacn}\right)^{3 \mathrm{a}}$ and $1,3,5$ triazacyclohexane $\left(\mathrm{R}_{3} \text { tach }\right)^{4}$ are also widely used as fac-coordinating $\mathrm{N}_{3}$-donor ligands in transition metal complexes, and more recently, monoanionic triazacyclononane $\left(\mathrm{R}_{2} \mathrm{tacn}^{-}\right)$ ligands have demonstrated their ability to support novel organometallic chemistry with main group and early transition metals. ${ }^{3 b}$

In the last few years, we have been involved in the study of the coordination chemistry of the trinuclear titanium imido-nitrido complex $\left[\left\{\mathrm{Ti}\left(\eta^{5}-\mathrm{C}_{5} \mathrm{Me}_{5}\right)(\mu-\mathrm{NH})\right\}_{3}\left(\mu_{3}-\mathrm{N}\right)\right]^{8,9}$ (1). Nitrido complexes of the early transition metals (Groups 4 and 5) remain rare in the literature. $^{10,11}$ This is possibly in part due to their polynuclear structures, which make extremely hard their characterization, and the lack of a systematic way of syntheses. The structure of complex 1 reveals a cyclic $\left[\mathrm{Ti}_{3}(\mu-\mathrm{NH})_{3}\right]$ system with three $\mathrm{NH}$ electron-donor imido groups and shows a strong resemblance to 1,4,7-triazacyclononane and, especially, 
1,3,5-triazacyclohexane ligands. This similarity prompted us to explore its potential application as a tridentate, preorganized, ${ }^{12}$ ligand towards different metal complexes and establish a rational strategy to prepare heterometallic nitrido complexes. This type of compounds might be of interest as building blocks in the synthesis of ternary metal nitride materials. $^{13}$

Our initial work with transition metal derivatives has shown that $\mathbf{1}$ is capable of acting as a neutral ligand through the basal $\mathrm{NH}$ groups, but later those imido groups can also be deprotonated to give monoanionic, dianionic and even trianionic forms of $\mathbf{1}$ depending on the metal and the other ligands present in the coordination sphere. ${ }^{11,14,15}$ In main-group chemistry, we have demonstrated the ability of $\mathbf{1}$ to coordinate metal cyclopentadienides and halides to afford stable adducts $\left[\mathrm{X}_{\mathrm{n}} \mathrm{M}\left\{\left(\mu_{3}-\mathrm{NH}\right)_{3} \mathrm{Ti}_{3}\left(\eta^{5}-\mathrm{C}_{5} \mathrm{Me}_{5}\right)_{3}\left(\mu_{3}-\mathrm{N}\right)\right\}\right] .{ }^{16}$ However, the analogous treatment of $\mathbf{1}$ with alkyl and amido derivatives of the alkali and alkaline-earth metals gave immediately the elimination of alkane or amine. Thus, we have isolated Group 1 metal complexes with edge-linked $\left[\left\{\mathrm{M}\left(\mu_{4}-\mathrm{N}\right)\left(\mu_{3}-\mathrm{NH}\right)_{2} \mathrm{Ti}_{3}\left(\eta^{5}-\mathrm{C}_{5} \mathrm{Me}_{5}\right)_{3}\left(\mu_{3}-\mathrm{N}\right)\right\}_{2}\right](\mathrm{M}=\mathrm{Li}, \mathrm{Na}, \mathrm{K})$ or corner-shared $\left[\mathrm{M}\left\{\left(\mu_{3}-\mathrm{N}\right)\left(\mu_{3}-\mathrm{NH}\right)_{5} \mathrm{Ti}_{6}\left(\eta^{5}-\mathrm{C}_{5} \mathrm{Me}_{5}\right)_{6}\left(\mu_{3}-\mathrm{N}\right)\right\}\right](\mathrm{M}=\mathrm{Na}, \mathrm{K}, \mathrm{Rb}, \mathrm{Cs})$ double cube structures upon depending on the reagents ratio $(1: 1$ or $2: 1)$ of 1 and $\left[\mathrm{MN}\left(\mathrm{SiMe}_{3}\right)_{2}\right] .{ }^{17,18}$ Group 2 alkyl and amido $\left[\mathrm{MR}_{2}\right]$ reagents react with 1 to give single-cube derivatives $\left[\mathrm{RM}\left\{\left(\mu_{3}-\mathrm{N}\right)\left(\mu_{3}-\right.\right.\right.$ $\left.\left.\mathrm{NH})_{2} \mathrm{Ti}_{3}\left(\eta^{5}-\mathrm{C}_{5} \mathrm{Me}_{5}\right)_{3}\left(\mu_{3}-\mathrm{N}\right)\right\}\right]$, but only the magnesium bis(trimethylsilyl)amido complex $\left[\left\{\left(\mathrm{Me}_{3} \mathrm{Si}\right)_{2} \mathrm{~N}\right\} \mathrm{Mg}\left\{\left(\mu_{3}-\mathrm{N}\right)\left(\mu_{3}-\mathrm{NH}\right)_{2} \mathrm{Ti}_{3}\left(\eta^{5}-\mathrm{C}_{5} \mathrm{Me}_{5}\right)_{3}\left(\mu_{3}-\mathrm{N}\right)\right\}\right]$ was stable in solution for long periods of time. In contrast, magnesium alkyls and derivatives bearing the heavier alkalineearth elements, undergo ligand redistribution reactions at room temperature to give cornershared complexes $\left[\mathrm{M}\left\{\left(\mu_{3}-\mathrm{N}\right)\left(\mu_{3}-\mathrm{NH}\right)_{2} \mathrm{Ti}_{3}\left(\eta^{5}-\mathrm{C}_{5} \mathrm{Me}_{5}\right)_{3}\left(\mu_{3}-\mathrm{N}\right)\right\}_{2}\right](\mathrm{M}=\mathrm{Mg}, \mathrm{Ca}, \mathrm{Sr}, \mathrm{Ba}){ }^{18,19}$

In view of these previous studies with s-block metals and as a logical continuation of our endeavor in the synthesis of new heterometallic nitrido complexes, we report here the results obtained in the treatment of $\mathbf{1}$ with analogous zinc(II) and copper(I) derivatives. The synthesis, 
structures and properties of the new compounds are discussed and compared with other zinc and cooper derivatives containing tridentate nitrogen-based ligands. ${ }^{20}$

\section{Experimental Section}

General Considerations. All manipulations were carried out under argon atmosphere using Schlenk line or glovebox techniques. Hexane was distilled from $\mathrm{Na} / \mathrm{K}$ alloy just before use. Toluene was freshly distilled from sodium. Dichloromethane was distilled from $\mathrm{P}_{2} \mathrm{O}_{5}$. NMR solvents were dried with $\mathrm{Na} / \mathrm{K}$ alloy $\left(\mathrm{C}_{6} \mathrm{D}_{6}, \mathrm{C}_{6} \mathrm{D}_{5} \mathrm{CD}_{3}\right)$ or calcium hydride $\left(\mathrm{CDCl}_{3}\right)$, and vacuum-distilled. Oven-dried glassware was repeatedly evacuated with a pumping system (ca. $1 \times 10^{-3}$ Torr) and subsequently filled with inert gas. Zinc(II) chloride (97\%), copper(I) chloride (99.99\%), copper(I) iodide (99.999\%), [ZnMe 2 (2.0 $\mathrm{M}$ in toluene), $\mathrm{PPh}_{3}$ and $\left[\mathrm{LiN}\left(\mathrm{SiMe}_{3}\right)_{2}\right]$ were purchased from Aldrich and used as received. $\left[\left\{\mathrm{Ti}\left(\eta^{5}-\mathrm{C}_{5} \mathrm{Me}_{5}\right)(\mu-\right.\right.$ $\left.\mathrm{NH})\}_{3}\left(\mu_{3}-\mathrm{N}\right)\right](\mathbf{1}),{ }^{8,9}\left[\mathrm{ZnR}_{2}\right]\left(\mathrm{R}=\mathrm{CH}_{2} \mathrm{SiMe}_{3},{ }^{21} \mathrm{CH}_{2} \mathrm{Ph},{ }^{22} \mathrm{~N}\left(\mathrm{SiMe}_{3}\right) 2,{ }^{23,24} \mathrm{C}_{5} \mathrm{H}_{4} \mathrm{SiMe}_{3},{ }^{25} \mathrm{C}_{5} \mathrm{H}_{5}{ }^{26}\right)$, $\left[\mathrm{LiCH}_{2} \mathrm{SiMe}_{3}\right],{ }^{27}\left[\mathrm{Li}\left(\mathrm{C}_{5} \mathrm{H}_{4} \mathrm{SiMe}_{3}\right)\right],{ }^{28}\left[\mathrm{Li}\left(\mathrm{C}_{5} \mathrm{H}_{5}\right)\right],{ }^{29}\left[\mathrm{Li}\left(\mathrm{C}_{9} \mathrm{H}_{7}\right)\right]^{30}$ and $\left[\left\{\mathrm{CuN}\left(\mathrm{SiMe}_{3}\right)_{2}\right\}_{4}\right]^{31,32}$ were prepared according to published procedures.

Samples for infrared spectroscopy were prepared as $\mathrm{KBr}$ pellets. ${ }^{1} \mathrm{H}$ and ${ }^{13} \mathrm{C}$ NMR spectra were recorded on a Varian Unity-300 and /or Unity-500 Plus spectrometer. Chemical shifts ( $\delta$, $\mathrm{ppm}$ ) are given relative to residual protons or to carbon of the solvent. Electron impact mass spectra were obtained at $70 \mathrm{eV}$. Microanalysis $(\mathrm{C}, \mathrm{H}, \mathrm{N})$ were performed in a Heraeus $\mathrm{CHN}$ O-Rapid or a Leco CHNS-932 microanalyzers.

Synthesis of $\left[\mathrm{Cl}_{2} \mathrm{Zn}\left\{\left(\mu_{3}-\mathbf{N H}\right)_{3} \mathbf{T i}_{3}\left(\eta^{5}-\mathrm{C}_{5} \mathbf{M e}_{5}\right)_{3}\left(\mu_{3}-\mathrm{N}\right)\right\}\right]$ (2): A $100 \mathrm{~mL}$ Schlenk flask was charged with $1(0.50 \mathrm{~g}, 0.82 \mathrm{mmol}), \mathrm{ZnCl}_{2}(0.11 \mathrm{~g}, 0.81 \mathrm{mmol})$ and toluene $(20 \mathrm{~mL})$. The reaction mixture was stirred at room temperature for $3 \mathrm{~h}$ to give a yellow solid and a brown solution. The solid was isolated by filtration onto a glass frit, washed with toluene $(5 \mathrm{~mL})$ and vacuum-dried to give 2 as a yellow powder $(0.41 \mathrm{~g}, 68 \%)$. IR $\left(\mathrm{KBr}, \mathrm{cm}^{-1}\right): 3363(\mathrm{w}), 3291$ (m), 2972 (w), 2908 (s), 2857 (m), 1618 (w), 1490 (w), 1429 (m), 1375 (m), 1260 (w), 1165 
(w), 1025 (w), 743 (s), $719(\mathrm{~s}), 704$ (s), 653 (vs), 624 (m), 606 (s), 549 (w), 532 (m), 468 (m), 419 (m). ${ }^{1} \mathrm{H}$ NMR $\left(\mathrm{CDCl}_{3}, 20{ }^{\circ} \mathrm{C}, \delta\right): 11.51$ (s broad, 3H; NH), 2.09 (s, 45H; $\left.\mathrm{C}_{5} \mathrm{Me}_{5}\right) .{ }^{13} \mathrm{C}\left\{{ }^{1} \mathrm{H}\right\}$ NMR $\left(\mathrm{CDCl}_{3}, 20{ }^{\circ} \mathrm{C}, \delta\right): 122.4\left(C_{5} \mathrm{Me}_{5}\right), 12.2\left(\mathrm{C}_{5} M e_{5}\right) . \mathrm{MS}(\mathrm{EI}, 70 \mathrm{eV}) \mathrm{m} / \mathrm{z}(\%): 709$ (2) [M$\mathrm{Cl}]^{+}, 608(1)\left[\mathrm{M}-\mathrm{ZnCl}_{2}\right]^{+}$. Anal. Calcd for $\mathrm{C}_{30} \mathrm{H}_{48} \mathrm{Cl}_{2} \mathrm{~N}_{4} \mathrm{Ti}_{3} \mathrm{Zn}\left(\mathrm{M}_{\mathrm{w}}=744.65\right)(\%): \mathrm{C} 48.39, \mathrm{H}$ 6.50, N 7.52. Found: C 48.22, H 6.60, N 7.26.

Reaction of 1 with $\left[\mathrm{Zn}\left(\mathrm{CH}_{2} \mathrm{SiMe}_{3}\right)_{2}\right]$ in a NMR tube scale experiment: A $5 \mathrm{~mm}$ ValvedNMR tube was charged with $1(0.010 \mathrm{~g}, 0.016 \mathrm{mmol}),\left[\mathrm{Zn}\left(\mathrm{CH}_{2} \mathrm{SiMe}_{3}\right)_{2}\right](0.004 \mathrm{~g}, 0.017$ mmol) and benzene- $\mathrm{d}_{6}(1.00 \mathrm{~mL})$. The resultant brown solution was monitored by NMR spectroscopy. After 10 minutes at room temperature, the spectra showed complete reaction and the corresponding complex $\left[\left(\mathrm{Me}_{3} \mathrm{SiCH}_{2}\right)_{2} \mathrm{Zn}\left\{\left(\mu_{3}-\mathrm{NH}\right)_{3} \mathrm{Ti}_{3}\left(\eta^{5}-\mathrm{C}_{5} \mathrm{Me}_{5}\right)_{3}\left(\mu_{3}-\mathrm{N}\right)\right\}\right]$ (4) was characterized by ${ }^{1} \mathrm{H}$ and ${ }^{13} \mathrm{C}\left\{{ }^{1} \mathrm{H}\right\}$ NMR spectroscopy. Complex 4 decomposed completely within hours at room temperature to give a red solution containing $\mathbf{5}$ and $\mathrm{SiMe}_{4}$ according to the NMR spectra.

NMR data for $\left[\left(\mathrm{Me}_{3} \mathrm{SiCH}_{2}\right)_{2} \mathrm{Zn}\left\{\left(\mu_{3}-\mathrm{NH}\right)_{3} \mathrm{Ti}_{3}\left(\eta^{5}-\mathrm{C}_{5} \mathrm{Me}_{5}\right)_{3}\left(\mu_{3}-\mathrm{N}\right)\right\}\right](4):{ }^{1} \mathrm{H} \mathrm{NMR}\left(\mathrm{C}_{6} \mathrm{D}_{6}, 20{ }^{\circ} \mathrm{C}\right.$,

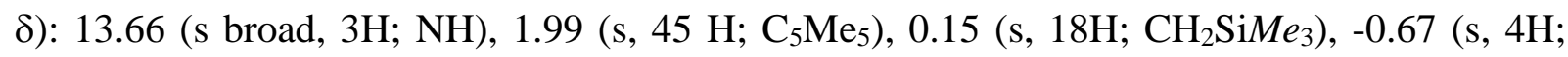
$\left.\mathrm{CH}_{2} \mathrm{SiMe}_{3}\right) .{ }^{13} \mathrm{C}\left\{{ }^{1} \mathrm{H}\right\}$ NMR $\left(\mathrm{C}_{6} \mathrm{D}_{6}, 20{ }^{\circ} \mathrm{C}, \delta\right): 117.5\left(\mathrm{C}_{5} \mathrm{Me}_{5}\right), 11.8\left(\mathrm{C}_{5} \mathrm{Me} e_{5}\right), 3.2\left(\mathrm{CH}_{2} \mathrm{SiMe}_{3}\right)$, $2.7\left(\mathrm{CH}_{2} \mathrm{SiMe}_{3}\right)$.

Synthesis of $\left[\left(\mathrm{Me}_{3} \mathrm{SiCH}_{2}\right) \mathrm{Zn}\left\{\left(\mu_{3}-\mathrm{N}\right)\left(\mu_{3}-\mathrm{NH}\right)_{2} \mathbf{T i}_{3}\left(\eta^{5}-\mathrm{C}_{5} \mathrm{Me}_{5}\right)_{3}\left(\mu_{3}-\mathrm{N}\right)\right\}\right]$ (5): Method A: A 100 mL Schlenk flask was charged with $1(0.30 \mathrm{~g}, 0.49 \mathrm{mmol}),\left[\mathrm{Zn}\left(\mathrm{CH}_{2} \mathrm{SiMe}_{3}\right)_{2}\right](0.12 \mathrm{~g}, 0.50$ $\mathrm{mmol})$ and toluene $(20 \mathrm{~mL})$. The reaction mixture was stirred at room temperature for $20 \mathrm{~h}$ to give an orange solution. After filtration, the volatile components were removed under reduced pressure to afford 5 as a red solid (0.34 g, 92\%). Method B: A $100 \mathrm{~mL}$ Schlenk flask was charged with $2(0.15 \mathrm{~g}, 0.20 \mathrm{mmol}),\left[\mathrm{LiCH}_{2} \mathrm{SiMe}_{3}\right](0.040 \mathrm{~g}, 0.42 \mathrm{mmol})$ and toluene $(25 \mathrm{~mL})$. The reaction mixture was stirred at room temperature for $20 \mathrm{~h}$ to give a red solution and a white precipitate. After filtration, the volatile components of the solution were removed under 
reduced pressure to afford 5 as a red solid $(0.11 \mathrm{~g}, 73 \%)$. IR $\left(\mathrm{KBr}, \mathrm{cm}^{-1}\right): 3363(\mathrm{w}), 2911$ (s), 2858 (s), 1491 (w), 1437 (m), 1376 (m), 1250 (w), 1239 (m), 1067 (w), 1024 (m), 937 (m), $857(\mathrm{~m}), 819$ (s), 787 (s), $722(\mathrm{vs}), 700$ (s), 675 (s), $616(\mathrm{~s}), 535(\mathrm{w}), 513(\mathrm{w}), 483(\mathrm{w}), 450$ (w), 426 (m). ${ }^{1} \mathrm{H}$ NMR $\left(\mathrm{C}_{6} \mathrm{D}_{6}, 20{ }^{\circ} \mathrm{C}, \delta\right.$ ): 10.55 (s broad, 2H; NH), 2.08 (s, 30H; $\mathrm{C}_{5} \mathrm{Me}_{5}$ ), 1.88 (s, $\left.15 \mathrm{H} ; \mathrm{C}_{5} \mathrm{Me}_{5}\right), 0.22$ (s, 9H; $\left.\mathrm{CH}_{2} \mathrm{SiMe}\right),-0.77$ (s, 2H; $\left.\mathrm{CH}_{2} \mathrm{SiMe}_{3}\right) .{ }^{13} \mathrm{C}\left\{{ }^{1} \mathrm{H}\right\} \mathrm{NMR}\left(\mathrm{C}_{6} \mathrm{D}_{6}, 20\right.$ $\left.{ }^{\circ} \mathrm{C}, \delta\right): 118.8,117.8\left(C_{5} \mathrm{Me}_{5}\right), 11.9,11.8\left(\mathrm{C}_{5} \mathrm{Me} e_{5}\right), 3.3\left(\mathrm{CH}_{2} \mathrm{SiMe}_{3}\right),-13.3\left(\mathrm{CH}_{2} \mathrm{SiMe}_{3}\right) . \mathrm{MS}(\mathrm{EI}$, $70 \mathrm{eV}) \mathrm{m} / \mathrm{z}(\%): 760(2)[\mathrm{M}]^{+}, 607(5)\left[\mathrm{M}-\mathrm{ZnCH}_{2} \mathrm{SiMe}_{3}\right]^{+}, 472(7)\left[\mathrm{M}-\mathrm{ZnCH}_{2} \mathrm{SiMe}_{3}-\mathrm{C}_{5} \mathrm{Me}_{5}\right]^{+}$. Anal. Calcd for $\mathrm{C}_{34} \mathrm{H}_{58} \mathrm{~N}_{4} \mathrm{SiTi}_{3} \mathrm{Zn}\left(\mathrm{M}_{\mathrm{w}}=759.96\right)(\%)$ : C 53.74, H 7.69, N 7.37. Found: C 53.62, H 7.54, N 6.69 .

Synthesis of $\left[\left(\mathrm{PhCH}_{2}\right) \mathrm{Zn}\left\{\left(\mu_{3}-\mathrm{N}\right)\left(\mu_{3}-\mathrm{NH}\right)_{2} \mathrm{Ti}_{3}\left(\eta^{5}-\mathrm{C}_{5} \mathrm{Me}_{5}\right)_{3}\left(\mu_{3}-\mathrm{N}\right)\right\}\right](6)$ : In a fashion similar to the preparation of 5, Method A, treatment of $\mathbf{1}(0.50 \mathrm{~g}, 0.82 \mathrm{mmol})$ with $\left[\mathrm{Zn}\left(\mathrm{CH}_{2} \mathrm{Ph}\right)_{2}\right](0.20 \mathrm{~g}$, $0.81 \mathrm{mmol})$ in toluene $(25 \mathrm{~mL})$ afforded 6 as a red solid $(0.57 \mathrm{~g}, 92 \%)$. IR $\left(\mathrm{KBr}, \mathrm{cm}^{-1}\right): 3361$ (w), 2908 (s), 2856 (s), 1593 (m), 1486 (m), 1429 (m), 1374 (m), 1204 (w), 1178 (w), 1057 (w), $1025(\mathrm{w}), 997(\mathrm{w}), 889(\mathrm{w}), 798(\mathrm{~m}), 749(\mathrm{~s}), 723$ (vs), $696(\mathrm{vs}), 664(\mathrm{~s}), 613(\mathrm{~m}), 539$ (w), 483 (w), 451 (w), 427 (s). ${ }^{1} \mathrm{H}$ NMR $\left(\mathrm{C}_{6} \mathrm{D}_{6}, 20{ }^{\circ} \mathrm{C}, \delta\right.$ ): 10.39 (s broad, 2H; NH), 7.20-6.89 (m, 5H; $\mathrm{CH}_{2} \mathrm{C}_{6} H_{5}$ ), 2.08 (s, 2H; $\left.\mathrm{CH}_{2} \mathrm{C}_{6} \mathrm{H}_{5}\right), 2.04$ (s, 30H; $\mathrm{C}_{5} \mathrm{Me}_{5}$ ), 1.81 (s, 15H; $\mathrm{C}_{5} \mathrm{Me}_{5}$ ). ${ }^{13} \mathrm{C}\left\{{ }^{1} \mathrm{H}\right\}$ NMR $\left(\mathrm{C}_{6} \mathrm{D}_{6}, 20{ }^{\circ} \mathrm{C}, \delta\right): 151.5,127.8,126.9,120.8\left(C_{6} \mathrm{H}_{5}\right), 119.1,118.0\left(C_{5} \mathrm{Me}_{5}\right), 14.1$

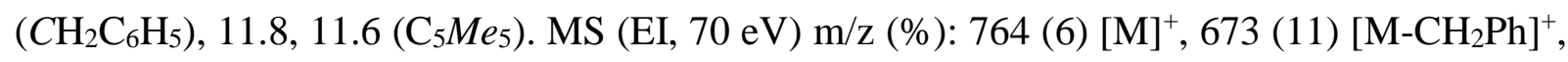
607 (21) $\left[\mathrm{M}-\mathrm{ZnCH}_{2} \mathrm{Ph}\right]^{+}$. Anal. Calcd for $\mathrm{C}_{37} \mathrm{H}_{54} \mathrm{~N}_{4} \mathrm{Ti}_{3} \mathrm{Zn}\left(\mathrm{M}_{\mathrm{w}}=763.87\right)(\%)$ : C 58.18, H 7.14, N 7.33. Found: C 57.87, H 7.42, N 7.05.

Synthesis of $\left[\operatorname{MeZn}\left\{(\mu 3-\mathrm{N})\left(\mu_{3}-\mathrm{NH}\right)_{2} \operatorname{Ti}_{3}\left(\eta^{5}-\mathrm{C}_{5} \mathrm{Me}_{5}\right)_{3}(\mu 3-\mathrm{N})\right\}\right](7)$ : In a fashion similar to the preparation of $\mathbf{6}$, treatment of $\mathbf{1}(1.00 \mathrm{~g}, 1.64 \mathrm{mmol})$ with [ $\left.\mathrm{ZnMe}_{2}\right](2.0 \mathrm{M}$ in toluene, $0.82 \mathrm{~mL}$, $1.64 \mathrm{mmol})$ in toluene $(50 \mathrm{~mL})$ afforded 7 as a red solid $(1.11 \mathrm{~g}, 98 \%)$. IR $\left(\mathrm{KBr}, \mathrm{cm}^{-1}\right): 3365$ (w), 2904 (s), 2856 (m), 2722 (w), 1489 (w), 1429 (m), 1375 (m), 1167 (w), 1066 (w), 1024 (w), $953(\mathrm{w}), 905$ (w), $783(\mathrm{~m}), 724$ (vs), 699 (vs), 664 (s), $616(\mathrm{~s}), 527(\mathrm{~m}), 484(\mathrm{w}), 452(\mathrm{w})$, 
428 (s). ${ }^{1} \mathrm{H}$ NMR $\left(\mathrm{C}_{6} \mathrm{D}_{6}, 20{ }^{\circ} \mathrm{C}, \delta\right): 10.66$ (s broad, $\left.2 \mathrm{H} ; \mathrm{NH}\right), 2.08$ (s, 30H; $\mathrm{C}_{5} \mathrm{Me}_{5}$ ), 1.86 (s, 15H; $\left.\mathrm{C}_{5} \mathrm{Me}_{5}\right),-0.37(\mathrm{~s}, 3 \mathrm{H} ; \mathrm{ZnMe}) .{ }^{13} \mathrm{C} \mathrm{NMR}\left(\mathrm{C}_{6} \mathrm{D}_{6}, 20{ }^{\circ} \mathrm{C}, \delta\right): 118.8\left(\mathrm{~m} ; C_{5} \mathrm{Me}_{5}\right), 117.8(\mathrm{~m} ;$ $\left.C_{5} \mathrm{Me}_{5}\right), 11.8\left(\mathrm{q},{ }^{1} \mathrm{~J}_{\mathrm{C}-\mathrm{H}}=125 \mathrm{~Hz} ; \mathrm{C}_{5} M e_{5}\right), 11.7\left(\mathrm{q},{ }^{1} \mathrm{~J}_{\mathrm{C}-\mathrm{H}}=126 \mathrm{~Hz} ; \mathrm{C}_{5} M e_{5}\right),-20.8\left(\mathrm{q},{ }^{1} \mathrm{~J}_{\mathrm{C}-\mathrm{H}}=121\right.$ Hz; ZnMe). MS (EI, 70 eV) m/z (\%): 688 (35) [M] $]^{+}, 673$ (4) [M-Me] $]^{+}, 607$ (27) [M-ZnMe $]^{+}$, 472 (25) $\left[\mathrm{M}-\mathrm{ZnMe}-\mathrm{C}_{5} \mathrm{Me}_{5}\right]^{+}, 337$ (27) $\left[\mathrm{M}-\mathrm{ZnMe}-2 \mathrm{C}_{5} \mathrm{Me}_{5}\right]^{+}$. Anal. Calcd for $\mathrm{C}_{31} \mathrm{H}_{50} \mathrm{~N}_{4} \mathrm{Ti}_{3} \mathrm{Zn}$ $\left(\mathrm{M}_{\mathrm{w}}=687.77\right)(\%)$ : C 54.14, H 7.33, N 8.15. Found: C 54.30, H 7.37, N 7.69.

Synthesis of $\left[\left\{\left(\mathrm{Me}_{3} \mathrm{Si}\right) \mathrm{H}_{4} \mathrm{C}_{5}\right\} \mathrm{Zn}\left\{\left(\mu_{3}-\mathrm{N}\right)\left(\mu_{3}-\mathrm{NH}\right)_{2} \mathrm{Ti}_{3}\left(\eta^{5}-\mathrm{C}_{5} \mathrm{Me}_{5}\right)_{3}\left(\mu_{3}-\mathrm{N}\right)\right\}\right]$ (8): In a fashion similar to the preparation of $\mathbf{5}$, Method A, treatment of $\mathbf{1}(0.30 \mathrm{~g}, 0.49 \mathrm{mmol})$ with $\left[\mathrm{Zn}\left\{\mathrm{C}_{5} \mathrm{H}_{4}\left(\mathrm{SiMe}_{3}\right)\right\}_{2}\right](0.17 \mathrm{~g}, 0.50 \mathrm{mmol})$ in toluene $(20 \mathrm{~mL})$ afforded $\mathbf{8}$ as a red solid $(0.39 \mathrm{~g}$, 98\%). Also using Method B, treatment of $2(0.30 \mathrm{~g}, 0.40 \mathrm{mmol})$ with $\left[\mathrm{Li}\left(\mathrm{C}_{5} \mathrm{H}_{4} \mathrm{SiMe}_{3}\right)\right](0.12 \mathrm{~g}$, $0.83 \mathrm{mmol})$ in toluene $(25 \mathrm{~mL})$ afforded 8 as a red solid $(0.25 \mathrm{~g}, 78 \%)$. IR $\left(\mathrm{KBr}, \mathrm{cm}^{-1}\right): 3361$ (w), 3344 (w), 3070 (w), 2948 (m), 2909 (s), 2860 (m), $1491(\mathrm{w}), 1430$ (m), 1375 (m), 1246 (m), 1145 (w), 1063 (w), 1043 (w), 1019 (m), 888 (m), 833 (s), 741 (vs), 720 (vs), 699 (vs), 613 (s), $532(\mathrm{~m}), 483(\mathrm{w}), 426(\mathrm{~m}) .{ }^{1} \mathrm{H}$ NMR $\left(\mathrm{C}_{6} \mathrm{D}_{6}, 20{ }^{\circ} \mathrm{C}, \delta\right): 9.61$ (s broad, 2H; NH), 6.88 (m, 2H; $\mathrm{C}_{5} \mathrm{H}_{2} \mathrm{H}_{2} \mathrm{SiMe}_{3}$ ), 6.67 (m, 2H; $\mathrm{C}_{5} \mathrm{H}_{2} \mathrm{H}_{2} \mathrm{SiMe}_{3}$ ), 2.06 (s, 30H; $\mathrm{C}_{5} \mathrm{Me}_{5}$ ), 1.85 (s, 15H; $\left.\mathrm{C}_{5} \mathrm{Me}_{5}\right), 0.19\left(\mathrm{~s}, 9 \mathrm{H} ; \mathrm{C}_{5} \mathrm{H}_{4}\left(\mathrm{Si}_{M} e_{3}\right)\right) .{ }^{13} \mathrm{C} \mathrm{NMR}\left(\mathrm{C}_{6} \mathrm{D}_{6}, 20{ }^{\circ} \mathrm{C}, \delta\right): 129.9\left(\mathrm{~m},{ }^{1} \mathrm{~J}_{\mathrm{C}-\mathrm{H}}=163 \mathrm{~Hz}\right.$; $\left.C_{4} \mathrm{H}_{4} \mathrm{CSiMe}_{3}\right), 121.5\left(\mathrm{~m},{ }^{1} \mathrm{~J}_{\mathrm{C}-\mathrm{H}}=158 \mathrm{~Hz} ; C_{4} \mathrm{H}_{4} \mathrm{CSiMe}_{3}\right), 119.5\left(\mathrm{~m} ; C_{5} \mathrm{Me}_{5}\right), 118.3\left(\mathrm{~m} ; C_{5} \mathrm{Me}_{5}\right)$, $72.1\left(\mathrm{~m} ; \mathrm{C}_{4} \mathrm{H}_{4} C \mathrm{CSiMe}_{3}\right), 12.0\left(\mathrm{q},{ }^{1} \mathrm{~J}_{\mathrm{C}-\mathrm{H}}=125 \mathrm{~Hz} ; \mathrm{C}_{5} M e_{5}\right), 11.8\left(\mathrm{q},{ }^{1} \mathrm{~J}_{\mathrm{C}-\mathrm{H}}=126 \mathrm{~Hz} ; \mathrm{C}_{5} M e_{5}\right), 0.3$ $\left(\mathrm{q},{ }^{1} \mathrm{~J}_{\mathrm{C}-\mathrm{H}}=117 \mathrm{~Hz} ; \mathrm{C}_{4} \mathrm{H}_{4} \mathrm{CSi} M e_{3}\right) . \mathrm{MS}(\mathrm{EI}, 70 \mathrm{eV}) \mathrm{m} / \mathrm{z}(\%): 810$ (2) [M] $]^{+}, 673$ (3) [M$\left.\mathrm{C}_{5} \mathrm{H}_{4} \mathrm{SiMe}_{3}\right]^{+}, 607$ (4) $\left[\mathrm{M}-\mathrm{ZnC}_{5} \mathrm{H}_{4} \mathrm{SiMe}_{3}\right]^{+}$. Anal. Calcd for $\mathrm{C}_{38} \mathrm{H}_{60} \mathrm{~N}_{4} \mathrm{SiTi}_{3} \mathrm{Zn}\left(\mathrm{M}_{\mathrm{w}}=810.02\right)$ (\%): C 56.35, H 7.46, N 6.92. Found: C 56.27, H 7.32, N 6.61.

Synthesis of $\left[\left\{\left(\mathrm{Me}_{3} \mathrm{Si}\right)_{2} \mathrm{~N}\right\} \mathrm{Zn}\left\{\left(\mu_{3}-\mathrm{N}\right)\left(\mu_{3}-\mathrm{NH}\right)_{2} \mathrm{Ti}_{3}\left(\eta^{5}-\mathrm{C}_{5} \mathrm{Me}_{5}\right)_{3}\left(\mu_{3}-\mathrm{N}\right)\right\}\right]$ (9): In a fashion similar to the preparation of $\mathbf{5}$, Method A, but using amber stained glassware, treatment of $\mathbf{1}$ $(1.00 \mathrm{~g}, 1.64 \mathrm{mmol})$ with $\left[\mathrm{Zn}\left\{\mathrm{N}\left(\mathrm{SiMe}_{3}\right)_{2}\right\}_{2}\right](0.63 \mathrm{~g}, 1.63 \mathrm{mmol})$ in toluene $(50 \mathrm{~mL})$ afforded 9 as a red solid $(1.19 \mathrm{~g}, 88 \%)$. Also through Method B but using amber stained glassware, 
treatment of $2(0.20 \mathrm{~g}, 0.27 \mathrm{mmol})$ with $\left[\mathrm{LiN}\left(\mathrm{SiMe}_{3}\right)_{2}\right](0.090 \mathrm{~g}, 0.54 \mathrm{mmol})$ in toluene (20 $\mathrm{mL})$ afforded 9 as a red solid $(0.15 \mathrm{~g}, 68 \%)$. IR $\left(\mathrm{KBr}, \mathrm{cm}^{-1}\right): 3383(\mathrm{w}), 3351(\mathrm{w}), 2909$ (s), 1491 (w), 1435 (m), 1377 (m), 1239 (s), 1066 (w), 992 (s), 885 (s), 830 (s), 720 (vs), 703 (vs), 665 (s), 615 (s), $530(\mathrm{w}), 480(\mathrm{w}), 445$ (w), 425 (m). ${ }^{1} \mathrm{H}$ NMR $\left(\mathrm{C}_{6} \mathrm{D}_{6}, 20{ }^{\circ} \mathrm{C}, \delta\right): 10.24$ (s broad, $2 \mathrm{H} ; \mathrm{NH}), 2.07\left(\mathrm{~s}, 30 \mathrm{H} ; \mathrm{C}_{5} \mathrm{Me}_{5}\right), 1.90\left(\mathrm{~s}, 15 \mathrm{H} ; \mathrm{C}_{5} \mathrm{Me}_{5}\right), 0.30\left(\mathrm{~s}, 18 \mathrm{H} ; \mathrm{N}\left(\mathrm{SiMe}_{3}\right)_{2}\right) .{ }^{13} \mathrm{C}\left\{{ }^{1} \mathrm{H}\right\}$ NMR $\left(\mathrm{C}_{6} \mathrm{D}_{6}, 20{ }^{\circ} \mathrm{C}, \delta\right): 119.6,118.4\left(C_{5} \mathrm{Me}_{5}\right), 12.1,12.0\left(\mathrm{C}_{5} M e_{5}\right), 6.7\left(\mathrm{SiMe}_{3}\right) . \mathrm{MS}(\mathrm{EI}, 70 \mathrm{eV})$ m/z (\%): 833 (2) $[\mathrm{M}]^{+}, 607$ (2) $\left[\mathrm{M}-\mathrm{ZnN}\left(\mathrm{SiMe}_{3}\right)_{2}\right]^{+}, 472(2)\left[\mathrm{M}-\mathrm{ZnN}\left(\mathrm{SiMe}_{3}\right)_{2}-\mathrm{C}_{5} \mathrm{Me}_{5}\right]^{+}$. Anal. Calcd for $\mathrm{C}_{36} \mathrm{H}_{65} \mathrm{~N}_{5} \mathrm{Si}_{2} \mathrm{Ti}_{3} \mathrm{Zn}\left(\mathrm{M}_{\mathrm{w}}=833.13\right)(\%)$ : C 51.90, H 7.86, N 8.41. Found: C 52.16, H 7.97, N 7.98.

Synthesis of $\left[\left\{\mathrm{Me} 3 \mathrm{Si}(\mathrm{H}) \mathrm{N}(\mathrm{Me})_{2} \mathrm{SiCH}_{2}\right\} \mathrm{Zn}\left\{\left(\mu_{3}-\mathrm{N}\right)\left(\mu_{3}-\mathrm{NH}\right)_{2} \mathrm{Ti}_{3}\left(\eta^{5}-\mathrm{C}_{5} \mathrm{Me}_{5}\right)_{3}\left(\mu_{3}-\mathrm{N}\right)\right\}\right]$ (10): A $150 \mathrm{~mL}$ ampoule (Teflon stopcock) was charged with 9 (1.21 g, $1.45 \mathrm{mmol})$, toluene $(50 \mathrm{~mL})$ and a magnetic stir bar. The orange solution was stirred under irradiation with a sun lamp (Ultra-Vitalux E27/ES OSRAM, $300 \mathrm{~W}$ ) for $20 \mathrm{~h}$. The resultant brown solution was filtered, and the volatile components were removed under reduced pressure to afford $\mathbf{1 0}$ as a brown solid (0.96 g, 79\%). IR (KBr, cm $\left.{ }^{-1}\right): 3365$ (m), 2948 (s), 2910 (s), 1494 (w), 1436 (m), 1376 (s), 1243 (s), 1168 (m), 1066 (w), 1023 (w), 922 (s), 838 (s), 800 (s), 774 (s), 723 (vs), 702 (vs), 678 (s), 616 (s), 529 (w), 483 (w), 451 (w), 427 (m). ${ }^{1} \mathrm{H}$ NMR $\left(\mathrm{C}_{6} \mathrm{D}_{6}, 20{ }^{\circ} \mathrm{C}, \delta\right): 10.54$ (s broad, 2H; NH), $2.08 \quad\left(\mathrm{~s}, \quad 30 \mathrm{H} ; \quad \mathrm{C}_{5} \mathrm{Me}_{5}\right), \quad 1.86 \quad\left(\mathrm{~s}, \quad 15 \mathrm{H} ; \quad \mathrm{C}_{5} \mathrm{Me}_{5}\right), \quad 0.30 \quad(\mathrm{~s}, \quad 6 \mathrm{H}$; $\left.\mathrm{CH}_{2} \mathrm{Si}(\mathrm{Me})_{2} \mathrm{~N}(\mathrm{H}) \mathrm{SiMe}_{3}\right), \quad 0.28 \quad\left(\mathrm{~s}, \quad 9 \mathrm{H} ; \quad \mathrm{CH}_{2} \mathrm{Si}(\mathrm{Me})_{2} \mathrm{~N}(\mathrm{H}) \mathrm{SiMe}_{3}\right), \quad-0.69 \quad(\mathrm{~s}, \quad 2 \mathrm{H} ;$ $\left.\mathrm{CH}_{2} \mathrm{Si}(\mathrm{Me})_{2} \mathrm{~N}(\mathrm{H}) \mathrm{SiMe}_{3}\right)$, not observed $\mathrm{CH}_{2} \mathrm{Si}(\mathrm{Me})_{2} \mathrm{~N}(H) \mathrm{SiMe}_{3} .{ }^{13} \mathrm{C} \mathrm{NMR}\left(\mathrm{C}_{6} \mathrm{D}_{6}, 20{ }^{\circ} \mathrm{C}, \delta\right)$ : $118.9\left(\mathrm{~m} ; C_{5} \mathrm{Me}_{5}\right), 117.9\left(\mathrm{~m} ; C_{5} \mathrm{Me}_{5}\right), 12.0\left(\mathrm{q},{ }^{1} \mathrm{~J}_{\mathrm{C}-\mathrm{H}}=125 \mathrm{~Hz} ; \mathrm{C}_{5} M e_{5}\right), 11.8\left(\mathrm{q},{ }^{1} \mathrm{~J}_{\mathrm{C}-\mathrm{H}}=126 \mathrm{~Hz}\right.$; $\left.\mathrm{C}_{5} M e_{5}\right), 5.6\left(\mathrm{q},{ }^{1} \mathrm{~J}_{\mathrm{C}-\mathrm{H}}=117 \mathrm{~Hz} ; \mathrm{CH}_{2} \mathrm{Si}(M e)_{2} \mathrm{~N}(\mathrm{H}) \mathrm{SiMe}_{3}\right), 3.2\left(\mathrm{q}, \quad{ }^{1} \mathrm{~J}_{\mathrm{C}-\mathrm{H}}=117 \mathrm{~Hz}\right.$; $\left.\mathrm{CH}_{2} \mathrm{Si}(\mathrm{Me})_{2} \mathrm{~N}(\mathrm{H}) \mathrm{Si} M e_{3}\right),-11.0\left(\mathrm{t},{ }^{1} \mathrm{~J}_{\mathrm{C}-\mathrm{H}}=115 \mathrm{~Hz} ; \mathrm{ZnCH}_{2} \mathrm{Si}(\mathrm{Me})_{2} \mathrm{~N}(\mathrm{H}) \mathrm{SiMe}_{3}\right) . \mathrm{MS}(\mathrm{EI}, 70 \mathrm{eV})$ m/z (\%): 833 (2) $[\mathrm{M}]^{+}, 607$ (4) $\left[\mathrm{M}-\mathrm{ZnCH}_{2} \mathrm{Si}(\mathrm{Me})_{2} \mathrm{NHSiMe}_{3}\right]^{+}$. Anal. Calcd for 
$\mathrm{C}_{36} \mathrm{H}_{65} \mathrm{~N}_{5} \mathrm{Si}_{2} \mathrm{Ti}_{3} \mathrm{Zn}\left(\mathrm{M}_{\mathrm{w}}=833.13\right)(\%): \mathrm{C} 51.90, \mathrm{H} 7.86, \mathrm{~N}$ 8.41. Found: C 52.33, H 7.96, N 7.52 .

Synthesis of $\left[\left(\mathrm{H}_{5} \mathrm{C}_{5}\right) \mathrm{Zn}\left\{\left(\mu_{3}-\mathrm{N}\right)\left(\mu_{3}-\mathbf{N H}\right)_{2} \mathbf{T i}_{3}\left(\eta^{5}-\mathrm{C}_{5} \mathrm{Me}_{5}\right)_{3}\left(\mu_{3}-\mathrm{N}\right)\right\}\right]$ (11): A $100 \mathrm{~mL}$ Schlenk flask was charged with $2(0.30 \mathrm{~g}, 0.40 \mathrm{mmol}),\left[\mathrm{Li}\left(\mathrm{C}_{5} \mathrm{H}_{5}\right)\right](0.060 \mathrm{~g}, 0.83 \mathrm{mmol})$ and toluene $(25 \mathrm{~mL})$. The reaction mixture was stirred at room temperature for $5 \mathrm{~h}$ to give a red solution and a white precipitate. After filtration, the volatile components were removed under reduced pressure to afford 11 as a red solid $(0.22 \mathrm{~g}, 73 \%)$. IR (KBr, $\left.\mathrm{cm}^{-1}\right): 3356$ (w), 3334 (w), 2909 (s), $2857(\mathrm{~m}), 1490(\mathrm{w}), 1431(\mathrm{~m}), 1375(\mathrm{~m}), 1261(\mathrm{w}), 1107(\mathrm{w}), 1067(\mathrm{w}), 1023(\mathrm{w}), 983$ (w), 781 (s), 730 (s), 702 (vs), 665 (s), 613 (s), 533 (m), 481 (w), 448 (m), 427 (s). ${ }^{1} \mathrm{H}$ NMR $\left(\mathrm{C}_{6} \mathrm{D}_{6}, 20{ }^{\circ} \mathrm{C}, \delta\right): 9.56$ (s broad, 2H; NH), 6.38 (s, 5H; $\left.\mathrm{C}_{5} \mathrm{H}_{5}\right), 2.04$ (s, 30H; $\left.\mathrm{C}_{5} \mathrm{Me}_{5}\right), 1.85$ (s, 15H; $\left.\mathrm{C}_{5} \mathrm{Me}_{5}\right) .{ }^{13} \mathrm{C}$ NMR $\left(\mathrm{C}_{6} \mathrm{D}_{6}, 20{ }^{\circ} \mathrm{C}, \delta\right): 119.6\left(\mathrm{~m}, C_{5} \mathrm{Me}_{5}\right), 118.3\left(\mathrm{~m}, C_{5} \mathrm{Me}_{5}\right), 108.8(\mathrm{~m}$, $\left.{ }^{1} \mathrm{~J}_{\mathrm{C}-\mathrm{H}}=160 \mathrm{~Hz} ; \mathrm{C}_{5} \mathrm{H}_{5}\right), 11.8\left(\mathrm{q},{ }^{1} \mathrm{~J}_{\mathrm{C}-\mathrm{H}}=125 \mathrm{~Hz} ; \mathrm{C}_{5} M e_{5}\right), 11.7\left(\mathrm{q},{ }^{1} \mathrm{~J}_{\mathrm{C}-\mathrm{H}}=126 \mathrm{~Hz} ; \mathrm{C}_{5} M e_{5}\right) . \mathrm{MS}$

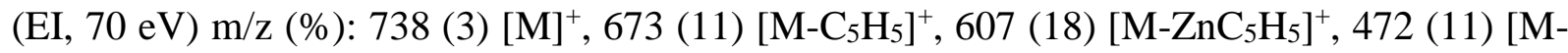
$\left.\mathrm{ZnC}_{5} \mathrm{H}_{5}-\mathrm{C}_{5} \mathrm{Me}_{5}\right]^{+}$. Anal. Calcd for $\mathrm{C}_{35} \mathrm{H}_{52} \mathrm{~N}_{4} \mathrm{Ti}_{3} \mathrm{Zn}\left(\mathrm{M}_{\mathrm{w}}=737.83\right)(\%)$ : C 56.98, H 7.10, N 7.59. Found: C 57.16, H 6.86, N 7.32.

Synthesis of $\left[\left(\mathrm{H}_{7} \mathrm{C}_{9}\right) \mathrm{Zn}\left\{\left(\mu_{3}-\mathrm{N}\right)\left(\mu_{3}-\mathbf{N H}\right)_{2} \mathbf{T i}_{3}\left(\eta^{5}-\mathrm{C}_{5} \mathbf{M e}_{5}\right)_{3}\left(\mu_{3}-\mathrm{N}\right)\right\}\right]$ (12): A $100 \mathrm{~mL}$ Schlenk flask was charged with $2(0.30 \mathrm{~g}, 0.40 \mathrm{mmol}),\left[\mathrm{Li}\left(\mathrm{C}_{9} \mathrm{H}_{7}\right)\right](0.10 \mathrm{~g}, 0.82 \mathrm{mmol})$ and toluene $(25$ $\mathrm{mL}$ ). The reaction mixture was stirred at room temperature for $3 \mathrm{~h}$ to give a red solution and a white precipitate. After filtration, the volatile components were removed under reduced pressure and the resultant solid was washed with hexane $(5 \mathrm{~mL})$ to afford $\mathbf{1 2}$ as a red powder (0.23 g, 72\%). IR (KBr, cm $\left.{ }^{-1}\right): 3352$ (w), 3055 (w), 2908 (m), 2856 (m), $1599(\mathrm{w}), 1491(\mathrm{w})$, $1431(\mathrm{~m}), 1375$ (m), 1323 (w), 1261 (w), 1230 (w), 1198 (w), $1161(\mathrm{w}), 1143(\mathrm{w}), 1063(\mathrm{w})$, $1024(\mathrm{w}), 992(\mathrm{w}), 924(\mathrm{w}), 887(\mathrm{w}), 865(\mathrm{w}), 809(\mathrm{~m}), 774(\mathrm{~m}), 735(\mathrm{~s}), 721(\mathrm{~s}), 699$ (vs), 664 (s), $614(\mathrm{~m}), 536(\mathrm{w}), 483(\mathrm{w}), 449(\mathrm{w}), 429(\mathrm{~m}) .{ }^{1} \mathrm{H}$ NMR $\left(\mathrm{C}_{6} \mathrm{D}_{6}, 20{ }^{\circ} \mathrm{C}, \delta\right): 9.85$ (s broad, 2H; $\mathrm{NH}), 7.67\left(\mathrm{~m}, 2 \mathrm{H} ; \mathrm{C}_{9} \mathrm{H}_{7}\right), 7.29\left(\mathrm{~m}, 2 \mathrm{H} ; \mathrm{C}_{9} \mathrm{H}_{7}\right), 7.05\left(\mathrm{t},{ }^{3} \mathrm{~J}=3 \mathrm{~Hz}, 1 \mathrm{H} ; \mathrm{C}_{9} \mathrm{H}_{7}\right), 5.63\left(\mathrm{~d},{ }^{3} \mathrm{~J}=3 \mathrm{~Hz}\right.$, 
$\left.2 \mathrm{H} ; \mathrm{C}_{9} \mathrm{H}_{7}\right), 2.03$ (s, 30H; $\left.\mathrm{C}_{5} \mathrm{Me}_{5}\right), 1.68$ (s, 15H; $\left.\mathrm{C}_{5} \mathrm{Me}_{5}\right) .{ }^{13} \mathrm{C}$ NMR $\left(\mathrm{C}_{6} \mathrm{D}_{6}, 20{ }^{\circ} \mathrm{C}, \delta\right): 144.8$ (s; $\left.C_{9} \mathrm{H}_{7}\right), 137.2\left(\mathrm{~d},{ }^{1} \mathrm{~J}_{\mathrm{C}-\mathrm{H}}=161 \mathrm{~Hz} ; C_{9} \mathrm{H}_{7}\right), 121.5\left(\mathrm{~m},{ }^{1} \mathrm{~J}_{\mathrm{C}-\mathrm{H}}=151 \mathrm{~Hz} ; C_{9} \mathrm{H}_{7}\right), 121.3\left(\mathrm{~m},{ }^{1} \mathrm{~J}_{\mathrm{C}-\mathrm{H}}=155\right.$ $\left.\mathrm{Hz} ; C_{9} \mathrm{H}_{7}\right), 119.6\left(\mathrm{~m}, C_{5} \mathrm{Me}_{5}\right), 118.3\left(\mathrm{~m}, C_{5} \mathrm{Me}_{5}\right), 82.2\left(\mathrm{~m},{ }^{1} \mathrm{~J}_{\mathrm{C}-\mathrm{H}}=152 \mathrm{~Hz} ; C_{9} \mathrm{H}_{7}\right), 11.8\left(\mathrm{q},{ }^{1} \mathrm{~J}_{\mathrm{C}-\mathrm{H}}\right.$ $\left.=125 \mathrm{~Hz} ; \mathrm{C}_{5} M e_{5}\right), 11.5\left(\mathrm{q},{ }^{1} \mathrm{~J}_{\mathrm{C}-\mathrm{H}}=125 \mathrm{~Hz} ; \mathrm{C}_{5} M e_{5}\right) . \mathrm{MS}(\mathrm{EI}, 70 \mathrm{eV}) \mathrm{m} / \mathrm{z}(\%): 788$ (2) $[\mathrm{M}]^{+}$, $673(24)\left[\mathrm{M}-\mathrm{C}_{9} \mathrm{H}_{7}\right]^{+}, 607$ (27) $\left[\mathrm{M}-\mathrm{ZnC}_{9} \mathrm{H}_{7}\right]^{+}, 472(24)\left[\mathrm{M}-\mathrm{ZnC}_{9} \mathrm{H}_{7}-\mathrm{C}_{5} \mathrm{Me}_{5}\right]^{+}, 337$ (41) [M$\left.\mathrm{ZnC}_{9} \mathrm{H}_{7}-2 \mathrm{C}_{5} \mathrm{Me}_{5}\right]^{+}$. Anal. Calcd for $\mathrm{C}_{39} \mathrm{H}_{54} \mathrm{~N}_{4} \mathrm{Ti}_{3} \mathrm{Zn}\left(\mathrm{M}_{\mathrm{w}}=787.89\right)(\%)$ : C 59.45, H 6.91, N 7.11. Found: C 59.59, H 6.72, N 7.20.

Synthesis of $\left[\mathbf{C l C u}\left\{\left(\mu_{3}-\mathbf{N H}\right)_{3} \mathbf{T i}_{3}\left(\eta^{5}-\mathbf{C}_{5} \mathbf{M e}_{5}\right)_{3}\left(\mu_{3}-\mathbf{N}\right)\right\}\right]$ (13): A $100 \mathrm{~mL}$ Schlenk flask was charged with 1 (0.70 g, $1.15 \mathrm{mmol}), \mathrm{CuCl}(0.11 \mathrm{~g}, 1.11 \mathrm{mmol})$ and toluene $(40 \mathrm{~mL})$. The reaction mixture was stirred at room temperature for $18 \mathrm{~h}$ to give a red solution. After filtration, the volatile components were removed under reduced pressure to give a red solid. The solid was washed with hexane $(15 \mathrm{~mL})$ and vacuum-dried to give $\mathbf{1 3}$ as a red powder (0.56 g, 72\%). IR (KBr, cm $\left.{ }^{-1}\right): 3362$ (m), 3219 (broad, m), 2909 (s), 2857 (m), 2723 (w), 1489 (w), $1450(\mathrm{~m}), 1428(\mathrm{~m}), 1377(\mathrm{~s}), 1067$ (w), 1025 (m), 797 (m), 739 (s), 668 (s), 645 (vs), 639 (vs), $520(\mathrm{w}), 476(\mathrm{w}), 430(\mathrm{~m}), 403(\mathrm{~m}) .{ }^{1} \mathrm{H}$ NMR $\left(\mathrm{C}_{6} \mathrm{D}_{6}, 20{ }^{\circ} \mathrm{C}, \delta\right): 11.70$ (s broad, 3H; NH), $1.87\left(\mathrm{~s}, 45 \mathrm{H} ; \mathrm{C}_{5} \mathrm{Me}_{5}\right) .{ }^{13} \mathrm{C}\left\{{ }^{1} \mathrm{H}\right\}$ NMR $\left(\mathrm{C}_{6} \mathrm{D}_{6}, 20^{\circ} \mathrm{C}, \delta\right): 119.4\left(C_{5} \mathrm{Me}_{5}\right), 11.6\left(\mathrm{C}_{5} M e_{5}\right) . \mathrm{MS}(\mathrm{EI}$, $70 \mathrm{eV}) \mathrm{m} / \mathrm{z}(\%): 706(1)[\mathrm{M}]^{+}, 671(4)[\mathrm{M}-\mathrm{Cl}]^{+}$. Anal. Calcd for $\mathrm{C}_{30} \mathrm{H}_{48} \mathrm{ClCuN}_{4} \mathrm{Ti}_{3}\left(\mathrm{M}_{\mathrm{w}}=\right.$ 707.34) (\%): C 50.94, H 6.84, N 7.92. Found: C 50.89, H 6.71, N 7.66.

Synthesis of $\left[\operatorname{ICu}\left\{\left(\mu_{3}-\mathrm{NH}\right)_{3} \operatorname{Ti}_{3}\left(\eta^{5}-\mathrm{C}_{5} \mathrm{Me}_{5}\right)_{3}\left(\mu_{3}-\mathrm{N}\right)\right\}\right]$ (14): In a fashion similar to the preparation of $\mathbf{1 3}$, treatment of $\mathbf{1}(0.50 \mathrm{~g}, 0.82 \mathrm{mmol})$ with $\mathrm{CuI}(0.16 \mathrm{~g}, 0.84 \mathrm{mmol})$ in toluene $(30 \mathrm{~mL})$ afforded 14 as a red solid $(0.43 \mathrm{~g}, 65 \%)$. IR (KBr, $\left.\mathrm{cm}^{-1}\right): 3363(\mathrm{~m}), 2906(\mathrm{~s}), 2857$ (m), $1488(\mathrm{w}), 1427(\mathrm{~m}), 1377(\mathrm{~m}), 1024(\mathrm{~m}), 746$ (s), 735 (s), 685 (s), 670 (s), 644 (vs), 628 (s), $522(\mathrm{w}), 479(\mathrm{w}), 435(\mathrm{~m}), 407(\mathrm{~m}) .{ }^{1} \mathrm{H}$ NMR $\left(\mathrm{C}_{6} \mathrm{D}_{6}, 20{ }^{\circ} \mathrm{C}, \delta\right): 11.68$ (s broad, 3H; NH), 1.87 (s, 45H; $\left.\mathrm{C}_{5} \mathrm{Me}_{5}\right) .{ }^{13} \mathrm{C}\left\{{ }^{1} \mathrm{H}\right\} \mathrm{NMR}\left(\mathrm{C}_{6} \mathrm{D}_{6}, 20{ }^{\circ} \mathrm{C}, \delta\right): 119.5\left(C_{5} \mathrm{Me}_{5}\right), 11.7\left(\mathrm{C}_{5} M e_{5}\right) . \mathrm{MS}(\mathrm{EI}$, 
$70 \mathrm{eV}$ ) m/z (\%): $671(23)[\mathrm{M}-\mathrm{I}]^{+}, 610(5)[\mathrm{M}-\mathrm{I}-\mathrm{Cu}]^{+}$. Anal. Calcd for $\mathrm{C}_{30} \mathrm{H}_{48} \mathrm{CuIN}_{4} \mathrm{Ti}_{3}\left(\mathrm{M}_{\mathrm{w}}=\right.$ 798.78) (\%): C 45.11, H 6.06, N 7.01. Found: C 45.41, H 5.77, N 6.63.

Synthesis of $\left[\left\{\mathrm{Cu}\left(\mu_{4}-\mathrm{N}\right)\left(\mu_{3}-\mathrm{NH}\right)_{2} \mathrm{Ti}_{3}\left(\eta^{5}-\mathrm{C}_{5} \mathrm{Me}_{5}\right)_{3}\left(\mu_{3}-\mathrm{N}\right)\right\}_{2}\right]$ (15): Method A: A solution of [ $\left.\mathrm{LiN}\left(\mathrm{SiMe}_{3}\right)_{2}\right](0.047 \mathrm{~g}, 0.28 \mathrm{mmol})$ in toluene $(5 \mathrm{~mL})$ was carefully added to $\mathbf{1 3}(0.20 \mathrm{~g}, 0.28$ $\mathrm{mmol})$ in toluene $(15 \mathrm{~mL})$. The system was allowed to react without any stirring for 6 days to give the precipitation of huge red crystals of $\mathbf{1 5}$ and a fine white powder. After elimination of the solution by decantation, the crystals were rinsed with several portions of toluene $(4 \times 5$ $\mathrm{mL})$ to remove the powder and characterized as $\mathbf{1 5} \cdot 2 \mathrm{C}_{7} \mathrm{H}_{8}(0.11 \mathrm{~g}, 51 \%)$. Method B: A 100 $\mathrm{mL}$ Carius tube was charged with $1(0.30 \mathrm{~g}, 0.49 \mathrm{mmol}),\left[\left\{\mathrm{CuN}\left(\mathrm{SiMe}_{3}\right)_{2}\right\}_{4}\right](0.11 \mathrm{~g}, 0.12$ mmol) and toluene $(30 \mathrm{~mL})$. The tube was flame sealed and heated at $150{ }^{\circ} \mathrm{C}$ for 6 days. The reaction mixture was allowed to cool to room temperature overnight to afford red crystals of 15. $2 \mathrm{C}_{7} \mathrm{H}_{8}(0.27 \mathrm{~g}, 73 \%)$. IR (KBr, cm $\left.{ }^{-1}\right): 3357$ (m), 2902 (s), 1604 (w), 1494 (m), 1431 (s), 1373 (s), 1080 (w), 1023 (m), 729 (vs), 671 (vs), 629 (vs), 617 (vs), 509 (s), 478 (w), 464 (m). Anal. Calcd for $\mathrm{C}_{74} \mathrm{H}_{110} \mathrm{Cu}_{2} \mathrm{~N}_{8} \mathrm{Ti}_{6}\left(\mathrm{M}_{\mathrm{w}}=1526.03\right)(\%)$ : C 58.24, H 7.26, N 7.34. Found: $\mathrm{C}$ 58.19, H 7.13, N 7.12.

Synthesis of $\left[\left(\mathbf{P h}_{3} \mathbf{P}\right) \mathrm{Cu}\left\{\left(\mu_{3}-\mathrm{N}\right)\left(\mu_{3}-\mathbf{N H}\right)_{2} \mathbf{T i}_{3}\left(\eta^{5}-\mathrm{C}_{5} \mathbf{M e}_{5}\right) 3\left(\mu_{3}-\mathrm{N}\right)\right\}\right]$ (16): A $100 \mathrm{~mL}$ Schlenk flask was charged with $13(0.30 \mathrm{~g}, 0.42 \mathrm{mmol}), \mathrm{PPh}_{3}(0.11 \mathrm{~g}, 0.42 \mathrm{mmol})$ and toluene (15 $\mathrm{mL})$. After stirring at room temperature for $10 \mathrm{~min}$., a solution of $\left[\mathrm{Li}\left\{\mathrm{N}\left(\mathrm{SiMe}_{3}\right)_{2}\right\}\right](0.070 \mathrm{~g}$, $0.42 \mathrm{mmol})$ in toluene $(5 \mathrm{~mL})$ was added. The reaction mixture was stirred at room temperature for $18 \mathrm{~h}$ to give an orange solution and a fine solid. After filtration, the volatile components were removed under reduced pressure to give $\mathbf{1 6}$ as an orange solid $(0.34 \mathrm{~g}, 87 \%)$. IR (KBr, cm $\left.{ }^{-1}\right): 3362(\mathrm{~m}), 3050$ (m), 2907 (s), 2855 (s), 1587 (w), 1573 (w), 1481 (m), 1434 (s), $1374(\mathrm{~m}), 1185$ (w), 1096 (m), 1026 (m), 997 (w), 744 (m), 707 (vs), 667 (vs), 613 (vs), $518(\mathrm{~s}), 492(\mathrm{~m}), 423(\mathrm{~m}), 387(\mathrm{~m}) .{ }^{1} \mathrm{H}$ NMR $\left(\mathrm{C}_{6} \mathrm{D}_{6}, 20{ }^{\circ} \mathrm{C}, \delta\right): 11.08$ (s broad, $\left.2 \mathrm{H} ; \mathrm{NH}\right), 7.59$ $\left(\mathrm{m}, 6 \mathrm{H} ; \mathrm{o}-\mathrm{C}_{6} \mathrm{H}_{5}\right), 7.15-7.10\left(\mathrm{~m}, 9 \mathrm{H} ; \mathrm{m}-\right.$ and $\left.\mathrm{p}-\mathrm{C}_{6} \mathrm{H}_{5}\right), 2.16\left(\mathrm{~s}, 30 \mathrm{H} ; \mathrm{C}_{5} \mathrm{Me}_{5}\right), 1.99(\mathrm{~s}, 15 \mathrm{H}$; 
$\left.\mathrm{C}_{5} \mathrm{Me}_{5}\right) .{ }^{13} \mathrm{C}\left\{{ }^{1} \mathrm{H}\right\}$ NMR $\left(\mathrm{C}_{6} \mathrm{D}_{6}, 20{ }^{\circ} \mathrm{C}, \delta\right): 135.5\left(\mathrm{~d},{ }^{1} \mathrm{~J}_{\mathrm{C}-\mathrm{P}}=33.7 \mathrm{~Hz}\right.$; ipso- $\left.\mathrm{C}_{6} \mathrm{H}_{5}\right), 134.3\left(\mathrm{~d},{ }^{2} \mathrm{~J}_{\mathrm{C}-\mathrm{P}}\right.$ $\left.=15.4 \mathrm{~Hz} ; \mathrm{o}-\mathrm{C}_{6} \mathrm{H}_{5}\right), 129.8\left(\mathrm{~d},{ }^{4} \mathrm{~J}_{\mathrm{C}-\mathrm{P}}=1.4 \mathrm{~Hz} ; \mathrm{p}-\mathrm{C}_{6} \mathrm{H}_{5}\right), 128.5\left(\mathrm{~d},{ }^{3} \mathrm{~J}_{\mathrm{C}-\mathrm{P}}=9.5 \mathrm{~Hz} ; \mathrm{m}-\mathrm{C}_{6} \mathrm{H}_{5}\right), 116.6$ $\left(C_{5} \mathrm{Me}_{5}\right), 115.8\left(C_{5} \mathrm{Me}_{5}\right), 11.9\left(\mathrm{C}_{5} M e_{5}\right), 11.8\left(\mathrm{C}_{5} M e_{5}\right) . \mathrm{MS}(\mathrm{EI}, 70 \mathrm{eV}) \mathrm{m} / \mathrm{z}(\%): 671$ (3) [M$\left.\mathrm{PPh}_{3}\right]^{+}, 608$ (1) $\left[\mathrm{M}-\mathrm{PPh}_{3}-\mathrm{Cu}\right]^{+}, 536$ (2) $\left[\mathrm{M}-\mathrm{PPh}_{3}-\mathrm{C}_{5} \mathrm{Me}_{5}\right]^{+}, 400$ (3) $\left[\mathrm{M}-\mathrm{PPh}_{3}-2 \mathrm{C}_{5} \mathrm{Me}_{5}\right]^{+}, 262$ (89) $\left[\mathrm{PPh}_{3}\right]^{+}, 183(100)\left[\mathrm{PPh}_{2}\right]^{+}, 108(40)[\mathrm{PPh}]^{+}$. Anal. Calcd for $\mathrm{C}_{48} \mathrm{H}_{62} \mathrm{CuN}_{4} \mathrm{PTi}_{3}\left(\mathrm{M}_{\mathrm{w}}=\right.$ 933.17) (\%): C 61.78, H 6.70, N 6.00. Found: C 61.62, H 6.54, N 5.36.

X-ray structure determination of 3, 8, 9 and 15. Crystals of complexes $3, \mathbf{8}, \mathbf{9}$ and 15 were grown as described in the results and discussion section, removed from the Schlenks and covered with a layer of a viscous perfluoropolyether (Fomblin $\left.{ }^{\circledR} Y\right)$. A suitable crystal was selected with the aid of a microscope, attached to a glass fiber, and immediately placed in the low temperature nitrogen stream of the diffractometer. The intensity data sets were collected at 200K on a Bruker-Nonius KappaCCD diffractometer equipped with an Oxford Cryostream 700 unit. Crystallographic data for all the complexes are presented in Table 1 (see page 34).

The structures were solved, using the WINGX package, ${ }^{33}$ by direct methods (SHELXS-97) and refined by least-squares against $\mathrm{F}^{2}$ (SHELXL-97). ${ }^{34}$ All non-hydrogen atoms were anisotropically refined while the hydrogen atoms were positioned geometrically and refined by using a riding model. Crystals of compound $\mathbf{1 5}$ were of poor quality and also presented disorder for the carbon atoms $\mathrm{C}(16)-\mathrm{C}(20)$ of the pentamethylcyclopentadienyl group linked to Ti(1), the PART tool and a free variable in the FVAR command of the SHELXL-97 program let to refine two positions for each carbon atom with $54.8 \%$ and $45.2 \%$ of occupancy respectively. The highest peak and hole found in the difference Fourier map of 1.58/-3.46 e $\AA^{-3}$ are located close to $\mathrm{Cu}(1), 1.03$ and $0.75 \AA ̊$ respectively. 


\section{Results and Discussion}

Azaheterometallocubanes of zinc. Treatment of $\left[\left\{\mathrm{Ti}\left(\eta^{5}-\mathrm{C}_{5} \mathrm{Me}_{5}\right)(\mu-\mathrm{NH})\right\}_{3}\left(\mu_{3}-\mathrm{N}\right)\right]$ (1) with anhydrous zinc dichloride (1 equiv) in toluene at room temperature affords the adduct $\left[\mathrm{Cl}_{2} \mathrm{Zn}\left\{\left(\mu_{3}-\mathrm{NH}\right)_{3} \mathrm{Ti}_{3}\left(\eta^{5}-\mathrm{C}_{5} \mathrm{Me}_{5}\right)_{3}\left(\mu_{3}-\mathrm{N}\right)\right\}\right]$ (2) as a yellow solid in $68 \%$ yield (Scheme 1$)$. Complex $\mathbf{2}$ is not soluble in aromatic solvents but exhibits a good solubility in chloroform or dichloromethane. ${ }^{1} \mathrm{H}$ and ${ }^{13} \mathrm{C}\left\{{ }^{1} \mathrm{H}\right\}$ NMR spectra of $\mathbf{2}$ in chloroform- $\mathrm{d}_{1}$ at room temperature show resonances for equivalent $\mathrm{NH}$ and $\eta^{5}-\mathrm{C}_{5} \mathrm{Me}_{5}$ groups. The $\mathrm{NH}$ resonance signal $(\delta=$ 11.51) in the ${ }^{1} \mathrm{H}$ NMR spectrum is shifted to higher field with respect to that found for $\mathbf{1}$ ( $\delta=$ 13.40), whereas the resonance signal for the ipso carbon of the pentamethylcyclopentadienyl groups $(\delta=122.4)$ in the ${ }^{13} \mathrm{C}\left\{{ }^{1} \mathrm{H}\right\}$ NMR spectrum is shifted downfield with respect to that found for $1(\delta=117.1)$. These data are similar to those recently reported for several maingroup metal adducts $\left[\mathrm{X}_{\mathrm{n}} \mathrm{M}\left\{\left(\mu_{3}-\mathrm{NH}\right)_{3} \mathrm{Ti}_{3}\left(\eta^{5}-\mathrm{C}_{5} \mathrm{Me}_{5}\right)_{3}\left(\mu_{3}-\mathrm{N}\right)\right\}\right],{ }^{16}$ and are consistent with a tridentate coordination of $\mathbf{1}$ to the zinc center or very fast exchange processes in solution.

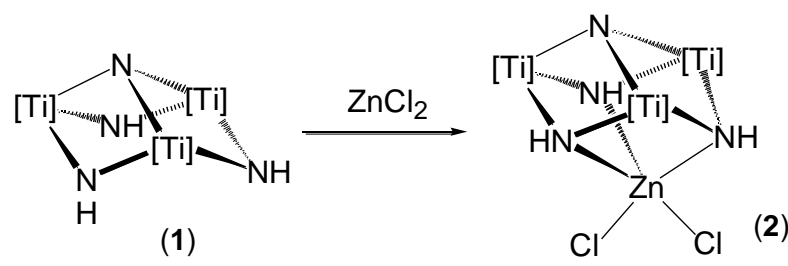

Scheme 1. Reaction of 1 with $\mathrm{ZnCl}_{2}$. [Ti] $=\operatorname{Ti}\left(\eta^{5}-\mathrm{C}_{5} \mathrm{Me}_{5}\right)$

Moreover, while the NMR data suggest a single-cube structure in solution, the insolubility of $\mathbf{2}$ in aromatic solvents might agree with a halide-bridged double cube structure in the solid state like that recently determined for the tin derivative $\left[\left\{\operatorname{ISn}\left(\mu_{3}-\mathrm{NH}\right)_{3} \mathrm{Ti}_{3}\left(\eta^{5}-\right.\right.\right.$ $\left.\left.\left.\mathrm{C}_{5} \mathrm{Me}_{5}\right)_{3}\left(\mu_{3}-\mathrm{N}\right)\right\}_{2}(\mu-\mathrm{I})_{2}\right] .{ }^{16 \mathrm{~b}}$ Mass spectrum $(\mathrm{EI}, 70 \mathrm{eV})$ shows the ion $[\mathrm{M}-\mathrm{Cl}]^{+}$as that of the higher relation mass/charge, suggesting a single-cube structure in the gas phase, but higher aggregates can not be ruled out. Attempts to grow suitable single crystals for a solid-state determination of 2 were failed, but a solution of 2 in dichloromethane at $-40{ }^{\circ} \mathrm{C}$ rendered a 
very small fraction of yellow crystals of the ammonia adduct $\left[\left(\mathrm{H}_{3} \mathrm{~N}\right) \mathrm{Cl}_{2} \mathrm{Zn}\left\{\left(\mu_{3}-\mathrm{NH}\right) \mathrm{Ti}_{3}\left(\eta^{5}-\right.\right.\right.$ $\left.\left.\left.\mathrm{C}_{5} \mathrm{Me}_{5}\right)_{3}(\mu-\mathrm{NH})_{2}\left(\mu_{3}-\mathrm{N}\right)\right\}\right]$ (3). Complex 3 could not be obtained in a preparative scale experiment since reactions of $\mathbf{2}$ with an excess of ammonia in dichloromethane gave immediately $\mathbf{1}$ and a fine white precipitate of $\left[\mathrm{ZnCl}_{2}\left(\mathrm{NH}_{3}\right)_{2}\right] \cdot{ }^{35,36}$ Furthermore, we did not observe any reaction between complex $\mathbf{1}$ and $\left[\mathrm{ZnCl}_{2}\left(\mathrm{NH}_{3}\right)_{2}\right]$ in dichloromethane or toluene even at high temperatures.

The X-ray crystal structure of $\left[\left(\mathrm{H}_{3} \mathrm{~N}\right) \mathrm{Cl}_{2} \mathrm{Zn}\left\{\left(\mu_{3}-\mathrm{NH}\right) \mathrm{Ti}_{3}\left(\eta^{5}-\mathrm{C}_{5} \mathrm{Me}_{5}\right)_{3}(\mu-\mathrm{NH})_{2}\left(\mu_{3}-\mathrm{N}\right)\right\}\right](3)$ is presented in Figure 1 and selected bond lengths and angles in Table 2. The solid-state structure reveals a distorted tetrahedral geometry for the zinc center, comprising two chloride, one ammonia ligand and one NH imido group with angles spanning 123.3(1)-100.8(2) ${ }^{\mathrm{o}}$. The

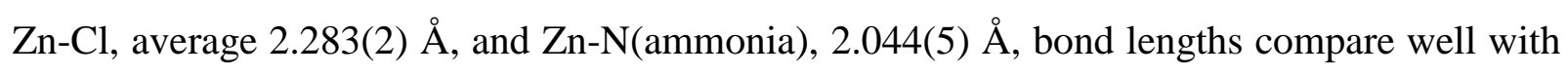
those reported for $\left[\mathrm{ZnCl}_{2}\left(\mathrm{NH}_{3}\right)_{2}\right]^{36}$ and other $\left[\mathrm{ZnCl}_{2} \text { (diamine) }\right]^{37}$ complexes. The triaza ligand is bound to zinc by one NH group with a zinc-nitrogen bond length, Zn-N(13) 2.046(5) $\AA$, very similar to that found for the ammonia ligand. Within the organometallic ligand, the average bond lengths and angles are similar to those determined in the free ligand $\mathbf{1}^{8}$

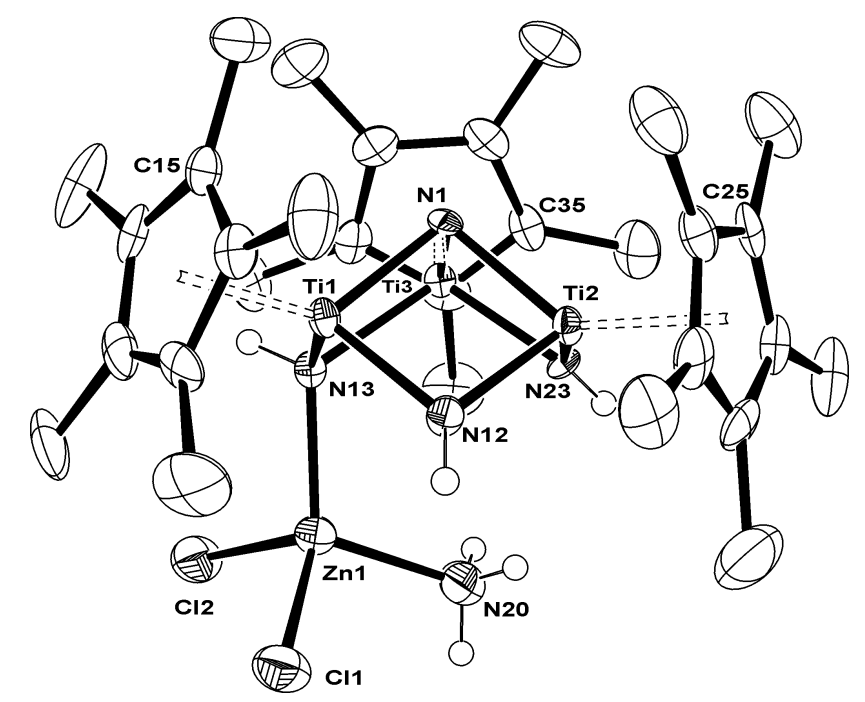

Figure 1. Perspective view of $\left[\left(\mathrm{H}_{3} \mathrm{~N}\right) \mathrm{Cl}_{2} \mathrm{Zn}\left\{\left(\mu_{3}-\mathrm{NH}\right) \mathrm{Ti}_{3}\left(\eta^{5}-\mathrm{C}_{5} \mathrm{Me}_{5}\right)_{3}(\mu-\mathrm{NH})_{2}\left(\mu_{3}-\mathrm{N}\right)\right\}\right](3)$ with thermal ellipsoids at the $50 \%$ probability level. The dichloromethane solvent molecule is omitted for clarity. 
Table 2. Selected bond lengths $(\AA)$ and angles $\left(^{\circ}\right)$ for 3.

\begin{tabular}{lrlr}
\hline $\mathrm{Zn}(1)-\mathrm{N}(13)$ & $2.046(5)$ & $\mathrm{Zn}(1)-\mathrm{N}(20)$ & $2.044(5)$ \\
$\mathrm{Zn}(1)-\mathrm{Cl}(1)$ & $2.274(2)$ & $\mathrm{Zn}(1)-\mathrm{Cl}(2)$ & $2.292(2)$ \\
$\mathrm{Ti}(1)-\mathrm{N}(13)$ & $2.009(4)$ & $\mathrm{Ti}(1)-\mathrm{N}(12)$ & $1.891(5)$ \\
$\mathrm{Ti}(1)-\mathrm{N}(1)$ & $1.908(4)$ & $\mathrm{Ti}(2)-\mathrm{N}(12)$ & $1.923(4)$ \\
$\mathrm{Ti}(2)-\mathrm{N}(23)$ & $1.927(4)$ & $\mathrm{Ti}(2)-\mathrm{N}(1)$ & $1.934(4)$ \\
$\mathrm{Ti}(3)-\mathrm{N}(13)$ & $2.009(4)$ & $\mathrm{Ti}(3)-\mathrm{N}(23)$ & $1.879(4)$ \\
$\mathrm{Ti}(3)-\mathrm{N}(1)$ & $1.922(4)$ & $\mathrm{Ti} \cdots \mathrm{Ti} \mathrm{av}$ & $2.815(1)$ \\
$\mathrm{N}(13)-\mathrm{Zn}(1)-\mathrm{N}(20)$ & $111.9(2)$ & $\mathrm{N}(13)-\mathrm{Zn}(1)-\mathrm{Cl}(1)$ & $123.3(1)$ \\
$\mathrm{N}(13)-\mathrm{Zn}(1)-\mathrm{Cl}(2)$ & $103.4(1)$ & $\mathrm{N}(20)-\mathrm{Zn}(1)-\mathrm{Cl}(1)$ & $100.8(2)$ \\
$\mathrm{N}(20)-\mathrm{Zn}(1)-\mathrm{Cl}(2)$ & $108.3(2)$ & $\mathrm{Cl}(1)-\mathrm{Zn}(1)-\mathrm{Cl}(2)$ & $108.7(1)$ \\
$\mathrm{Ti}(1)-\mathrm{N}(1)-\mathrm{Ti}(3)$ & $95.9(2)$ & $\mathrm{Ti}(1)-\mathrm{N}(1)-\mathrm{Ti}(2)$ & $93.9(2)$ \\
$\mathrm{Ti}(3)-\mathrm{N}(1)-\mathrm{Ti}(2)$ & $93.0(2)$ & $\mathrm{Ti}(1)-\mathrm{N}(12)-\mathrm{Ti}(2)$ & $94.8(2)$ \\
$\mathrm{Ti}(3)-\mathrm{N}(13)-\mathrm{Ti}(1)$ & $90.1(2)$ & $\mathrm{Ti}(3)-\mathrm{N}(13)-\mathrm{Zn}(1)$ & $120.0(2)$ \\
$\mathrm{Ti}(1)-\mathrm{N}(13)-\mathrm{Zn}(1)$ & $113.0(2)$ & $\mathrm{Ti}(3)-\mathrm{N}(23)-\mathrm{Ti}(2)$ & $94.6(2)$ \\
$\mathrm{N}(12)-\mathrm{Ti}(1)-\mathrm{N}(1)$ & $86.2(2)$ & $\mathrm{N}(12)-\mathrm{Ti}(1)-\mathrm{N}(13)$ & $103.6(2)$ \\
$\mathrm{N}(1)-\mathrm{Ti}(1)-\mathrm{N}(13)$ & $87.0(2)$ & $\mathrm{N}(12)-\mathrm{Ti}(2)-\mathrm{N}(23)$ & $107.3(2)$ \\
$\mathrm{N}(12)-\mathrm{Ti}(2)-\mathrm{N}(1)$ & $84.6(2)$ & $\mathrm{N}(23)-\mathrm{Ti}(2)-\mathrm{N}(1)$ & $85.0(2)$ \\
$\mathrm{N}(23)-\mathrm{Ti}(3)-\mathrm{N}(1)$ & $86.7(2)$ & $\mathrm{N}(23)-\mathrm{Ti}(3)-\mathrm{N}(13)$ & $105.3(2)$ \\
$\mathrm{N}(1)-\mathrm{Ti}(3)-\mathrm{N}(13)$ & $86.7(2)$ & & \\
\hline
\end{tabular}

Treatment of 1 with one equivalent of the zinc alkyl, trimethylsilylcyclopentadienyl or amido, complexes $\left[\mathrm{ZnR}_{2}\right]$ in toluene at room temperature leads to the cube-type derivatives $\left[\operatorname{RZn}\left\{\left(\mu_{3}-\mathrm{N}\right)\left(\mu_{3}-\mathrm{NH}\right)_{2} \mathrm{Ti}_{3}\left(\eta^{5}-\mathrm{C}_{5} \mathrm{Me}_{5}\right)_{3}\left(\mu_{3}-\mathrm{N}\right)\right\}\right]\left[\mathrm{R}=\mathrm{CH}_{2} \mathrm{SiMe}_{3} \quad(\mathbf{5}), \mathrm{CH}_{2} \mathrm{Ph} \quad(\mathbf{6}), \mathrm{Me}(\mathbf{7})\right.$, $\left.\mathrm{C}_{5} \mathrm{H}_{4} \mathrm{SiMe}_{3}(\mathbf{8}), \mathrm{N}\left(\mathrm{SiMe}_{3}\right)_{2}(\mathbf{9})\right]$ via alkane/trimethylsilylcyclopentadiene/amine elimination (Scheme 2). However, the analogous treatment of 1 with dicyclopentadienylzinc $\left[\mathrm{Zn}\left(\mathrm{C}_{5} \mathrm{H}_{5}\right)_{2}\right]^{26}$ gave the initial products unaltered, probably due to the insolubility of the zinc reagent in the reaction solvent.

The reactions most likely proceed via adducts similar to $\mathbf{2}$, but only the bis(trimethylsilylmethyl)zinc derivative $\left[\left(\mathrm{Me}_{3} \mathrm{SiCH}_{2}\right)_{2} \mathrm{Zn}\left\{\left(\mu_{3}-\mathrm{NH}\right)_{3} \mathrm{Ti}_{3}\left(\eta^{5}-\mathrm{C}_{5} \mathrm{Me}_{5}\right)_{3}\left(\mu_{3}-\mathrm{N}\right)\right\}\right](4)$ was stable for some minutes and clearly identified following the reaction course by ${ }^{1} \mathrm{H}$ and ${ }^{13} \mathrm{C}\left\{{ }^{1} \mathrm{H}\right\}$ NMR spectroscopy. The spectra at room temperature of the reaction mixture in benzene- $\mathrm{d}_{6}$ after $10 \mathrm{~min}$. show shifted resonances with respect to those of the starting materials for equivalent $\mathrm{NH}, \eta^{5}-\mathrm{C}_{5} \mathrm{Me}_{5}$, and $\mathrm{CH}_{2} \mathrm{SiMe}_{3}$ groups. After some hours at room temperature, 
the spectra revealed complete consumption of $\mathbf{4}$ to give complex $\mathbf{5}$ and the corresponding equivalent of tetramethylsilane.

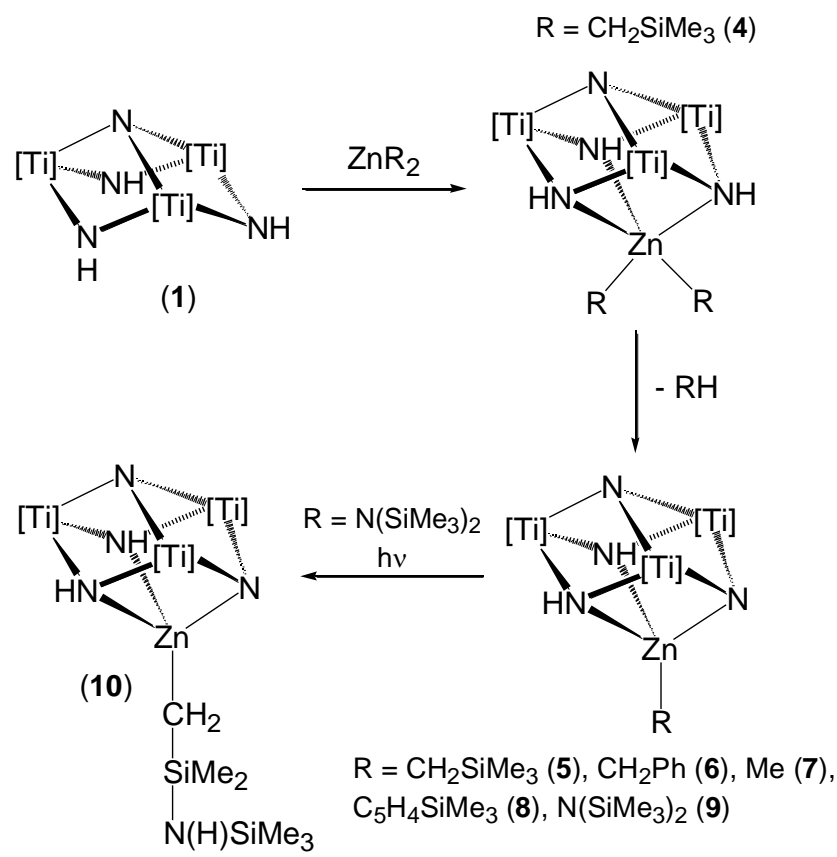

Scheme 2. Reactions of 1 with $\mathrm{ZnR}_{2}$. [Ti] $=\mathrm{Ti}\left(\eta^{5}-\mathrm{C}_{5} \mathrm{Me}_{5}\right)$

Complexes 5-9 were obtained in good yields (88-98\%) as red solids, which are very soluble in hexane or toluene. These compounds were characterized by spectral and analytical methods, as well as by X-ray crystal structure determinations for $\mathbf{8}$ and $\mathbf{9}$. Mass spectra (EI, 70 $\mathrm{eV}$ ) show the expected molecular peaks suggesting a single-cube aggregation in the gas phase. ${ }^{1} \mathrm{H}$ and ${ }^{13} \mathrm{C}$ NMR spectra in benzene- $\mathrm{d}_{6}$ at room temperature show resonance signals for two $\eta^{5}-\mathrm{C}_{5} \mathrm{Me}_{5}$ groups in a 2:1 ratio, one $\mathrm{Zn}-\mathrm{R}$ fragment and a broad signal for the $\mathrm{NH}$ groups. These NMR data are consistent with a $\mathrm{C}_{\mathrm{s}}$ symmetry in solution and tetrahedral geometries for the zinc centers, in a similar way to those recently reported for the analogous magnesium complexes $\quad\left[\operatorname{RMg}\left\{\left(\mu_{3}-\mathrm{N}\right)\left(\mu_{3}-\mathrm{NH}\right)_{2} \mathrm{Ti}_{3}\left(\eta^{5}-\mathrm{C}_{5} \mathrm{Me}_{5}\right)_{3}\left(\mu_{3}-\mathrm{N}\right)\right\}\right]^{19} \quad$ and $\quad$ several tris(pyrazolyl)hydroboratozinc derivatives. ${ }^{38}$

In order to confirm the proposed structures in the Scheme 2 and establish unambiguously the coordination mode of the trimethylsilylcyclopentadienyl ligand in $\mathbf{8}, \mathrm{X}$-ray crystallographic studies were undertaken for crystals of $\mathbf{8} \cdot \mathrm{C}_{7} \mathrm{H}_{8}$ and 9 grown in saturated 
toluene solutions. The X-ray structures are presented in Figures 2 and 3, while selected bond and angles are shown in Tables 3 and 4.

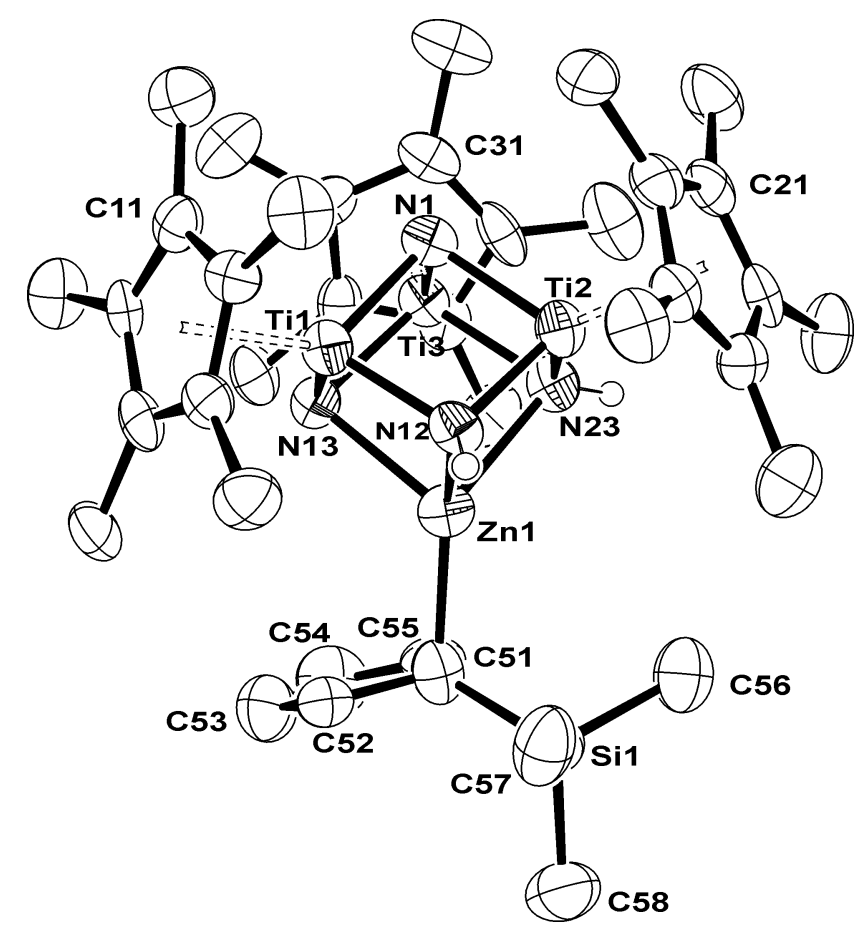

Figure 2. Perspective view of $\left[\left\{\left(\mathrm{Me} \mathrm{S}_{3}\right) \mathrm{H}_{4} \mathrm{C}_{5}\right\} \mathrm{Zn}\left\{\left(\mu_{3}-\mathrm{N}\right)\left(\mu_{3}-\mathrm{NH}\right)_{2} \mathrm{Ti}_{3}\left(\eta^{5}-\mathrm{C}_{5} \mathrm{Me}_{5}\right)_{3}\left(\mu_{3}-\mathrm{N}\right)\right\}\right](\mathbf{8})$ with thermal ellipsoids at the $50 \%$ probability level. The toluene solvent molecule is omitted for clarity.

Table 3. Selected bond lengths $(\AA)$ and angles $\left(^{\circ}\right)$ for $\mathbf{8}$.

\begin{tabular}{lrlr}
\hline $\mathrm{Zn}(1)-\mathrm{N}(12)$ & $2.148(8)$ & $\mathrm{Zn}(1)-\mathrm{N}(13)$ & $2.126(8)$ \\
$\mathrm{Zn}(1)-\mathrm{N}(23)$ & $2.147(8)$ & $\mathrm{Zn}(1)-\mathrm{C}(51)$ & $2.031(11)$ \\
$\mathrm{Zn}(1) \cdots \mathrm{C}(52)$ & $2.753(11)$ & $\mathrm{Zn}(1) \cdots \mathrm{C}(53)$ & $3.532(14)$ \\
$\mathrm{Zn}(1) \cdots \mathrm{C}(54)$ & $3.482(15)$ & $\mathrm{Zn}(1) \cdots \mathrm{C}(55)$ & $2.686(12)$ \\
$\mathrm{C}(51)-\mathrm{C}(52)$ & $1.461(14)$ & $\mathrm{C}(51)-\mathrm{C}(55)$ & $1.457(14)$ \\
$\mathrm{C}(52)-\mathrm{C}(53)$ & $1.372(16)$ & $\mathrm{C}(53)-\mathrm{C}(54)$ & $1.391(18)$ \\
$\mathrm{C}(54)-\mathrm{C}(55)$ & $1.376(16)$ & $\mathrm{C}(51)-\mathrm{Si}(1)$ & $1.855(11)$ \\
$\mathrm{N}(1)-\mathrm{Ti}(3)$ & $1.895(8)$ & $\mathrm{N}(1)-\mathrm{Ti}(1)$ & $1.922(8)$ \\
$\mathrm{N}(1)-\mathrm{Ti}(2)$ & $1.949(8)$ & $\mathrm{N}(12)-\mathrm{Ti}(2)$ & $1.857(7)$ \\
$\mathrm{N}(12)-\mathrm{Ti}(1)$ & $1.853(8)$ & $\mathrm{N}(13)-\mathrm{Ti}(3)$ & $1.961(8)$ \\
$\mathrm{N}(13)-\mathrm{Ti}(1)$ & $1.968(8)$ & $\mathrm{N}(23)-\mathrm{Ti}(2)$ & $1.946(8)$ \\
$\mathrm{N}(23)-\mathrm{Ti}(3)$ & $1.958(8)$ & $\mathrm{Ti} \cdots \mathrm{Ti}$ av & $2.817(2)$ \\
$\mathrm{N}(12)-\mathrm{Zn}(1)-\mathrm{N}(13)$ & $86.2(3)$ & $\mathrm{N}(12)-\mathrm{Zn}(1)-\mathrm{N}(23)$ & $84.7(3)$ \\
$\mathrm{N}(13)-\mathrm{Zn}(1)-\mathrm{N}(23)$ & $87.1(3)$ & $\mathrm{N}(12)-\mathrm{Zn}(1)-\mathrm{C}(51)$ & $129.0(4)$ \\
$\mathrm{N}(13)-\mathrm{Zn}(1)-\mathrm{C}(51)$ & $122.1(4)$ & $\mathrm{N}(23)-\mathrm{Zn}(1)-\mathrm{C}(51)$ & $132.8(4)$ \\
$\mathrm{Zn}(1)-\mathrm{C}(51)-\mathrm{Si}(1)$ & $107.9(5)$ & $\mathrm{Zn}(1)-\mathrm{C}(51)-\mathrm{Cp}$ & $108.8(6)$ \\
$\mathrm{Si}(1)-\mathrm{C}(51)-\mathrm{Cp}$ & $143.5(7)$ & $\mathrm{Ti}(3)-\mathrm{N}(1)-\mathrm{Ti}(1)$ & $96.3(4)$
\end{tabular}




\begin{tabular}{llll} 
Ti(3)-N(1)-Ti(2) & $95.1(3)$ & $\mathrm{Ti}(1)-\mathrm{N}(1)-\mathrm{Ti}(2)$ & $91.4(3)$ \\
$\mathrm{Ti}(2)-\mathrm{N}(12)-\mathrm{Ti}(1)$ & $96.7(3)$ & $\mathrm{Ti}(2)-\mathrm{N}(12)-\mathrm{Zn}(1)$ & $89.1(3)$ \\
$\mathrm{Ti}(1)-\mathrm{N}(12)-\mathrm{Zn}(1)$ & $87.9(3)$ & $\mathrm{Ti}(3)-\mathrm{N}(13)-\mathrm{Ti}(1)$ & $92.7(3)$ \\
$\mathrm{Ti}(3)-\mathrm{N}(13)-\mathrm{Zn}(1)$ & $87.9(3)$ & $\mathrm{Ti}(1)-\mathrm{N}(13)-\mathrm{Zn}(1)$ & $85.6(3)$ \\
$\mathrm{Ti}(2)-\mathrm{N}(23)-\mathrm{Ti}(3)$ & $93.2(3)$ & $\mathrm{Ti}(2)-\mathrm{N}(23)-\mathrm{Zn}(1)$ & $86.8(3)$ \\
$\mathrm{Ti}(3)-\mathrm{N}(23)-\mathrm{Zn}(1)$ & $87.4(3)$ & $\mathrm{N}(12)-\mathrm{Ti}(1)-\mathrm{N}(1)$ & $86.4(3)$ \\
$\mathrm{N}(12)-\mathrm{Ti}(1)-\mathrm{N}(13)$ & $99.7(3)$ & $\mathrm{N}(1)-\mathrm{Ti}(1)-\mathrm{N}(13)$ & $84.9(3)$ \\
$\mathrm{N}(12)-\mathrm{Ti}(2)-\mathrm{N}(1)$ & $85.5(3)$ & $\mathrm{N}(12)-\mathrm{Ti}(2)-\mathrm{N}(23)$ & $99.0(3)$ \\
$\mathrm{N}(1)-\mathrm{Ti}(2)-\mathrm{N}(23)$ & $85.2(3)$ & $\mathrm{N}(1)-\mathrm{Ti}(3)-\mathrm{N}(23)$ & $86.4(3)$ \\
$\mathrm{N}(1)-\mathrm{Ti}(3)-\mathrm{N}(13)$ & $85.8(3)$ & $\mathrm{N}(23)-\mathrm{Ti}(3)-\mathrm{N}(13)$ & $97.4(3)$ \\
\hline $\mathrm{Cp}$
\end{tabular}

$\mathrm{Cp}=$ centroid of the $\mathrm{C}(51)-\mathrm{C}(55)$ ring

Compound 8 crystallizes with one toluene solvent molecule per cube-type complex. The structure shows a cube-type $\left[\mathrm{ZnTi}_{3} \mathrm{~N}_{4}\right]$ core with angles M-N-M and N-M-N spanning 85.2(3)$99.7(3)^{\circ}$. The coordination sphere about the zinc atom is best described as distorted tetrahedral, comprising three nitrogen atoms of the tridentate organometallic ligand " $\left\{\left(\mu_{3}-\mathrm{N}\right)\left(\mu_{3^{-}}\right.\right.$ $\left.\mathrm{NH})_{2} \mathrm{Ti}_{3}\left(\eta^{5}-\mathrm{C}_{5} \mathrm{Me}_{5}\right)_{3}\left(\mu_{3}-\mathrm{N}\right)\right\}^{-”}$ and one carbon atom of the $\mathrm{C}_{5} \mathrm{H}_{4} \mathrm{SiMe}_{3}$ group, with angles N$\mathrm{Zn}-\mathrm{N}$ 86.0(3) av. and $\mathrm{C}(51)-\mathrm{Zn}-\mathrm{N} 128.0(4)^{\circ}$ av. The zinc-nitrogen bond lengths, average 2.140(8) $\AA$, are slightly longer than that found for complex $\mathbf{3}$ but closer to those reported for several tris(pyrazolyl)hydroborates ${ }^{38 a, 39}$ where the zinc atom is also coordinated to three nitrogen atoms. The distance from the silyl-bearing carbon atom $\mathrm{C}(51)$ to the zinc atom [2.031(11) $\AA$ ], typical for zinc-carbon covalent bonds, ${ }^{40}$ is clearly shorter than those to the other carbon atoms of the trimethylsilylcyclopentadienyl ring, as can be seen in Table 3 , in a fashion similar to those found in $\left[\mathrm{Zn}\left(\mathrm{C}_{5} \mathrm{Me}_{4} \mathrm{SiMe}_{3}\right)_{2}\right]^{41}$ and $\left[\mathrm{Zn}\left(\mathrm{C}_{5} \mathrm{H}_{4} \mathrm{SiMe}_{3}\right)_{2}\right]{ }^{25 \mathrm{a}}$ confirming an $\eta^{1}(\sigma)$ coordination mode. Moreover, the $\mathrm{Zn}-\mathrm{C}(51)-\mathrm{Si}$ and $\mathrm{Zn}-\mathrm{C}(51)$-ring angles [107.9(5) and $108.8(6)^{\circ}$, respectively] are very close to the ideal $109.5^{\circ}$ value for a tetrahedral geometry, suggesting an $\mathrm{sp}^{3}$ hybridization for $\mathrm{C}(51)$. 


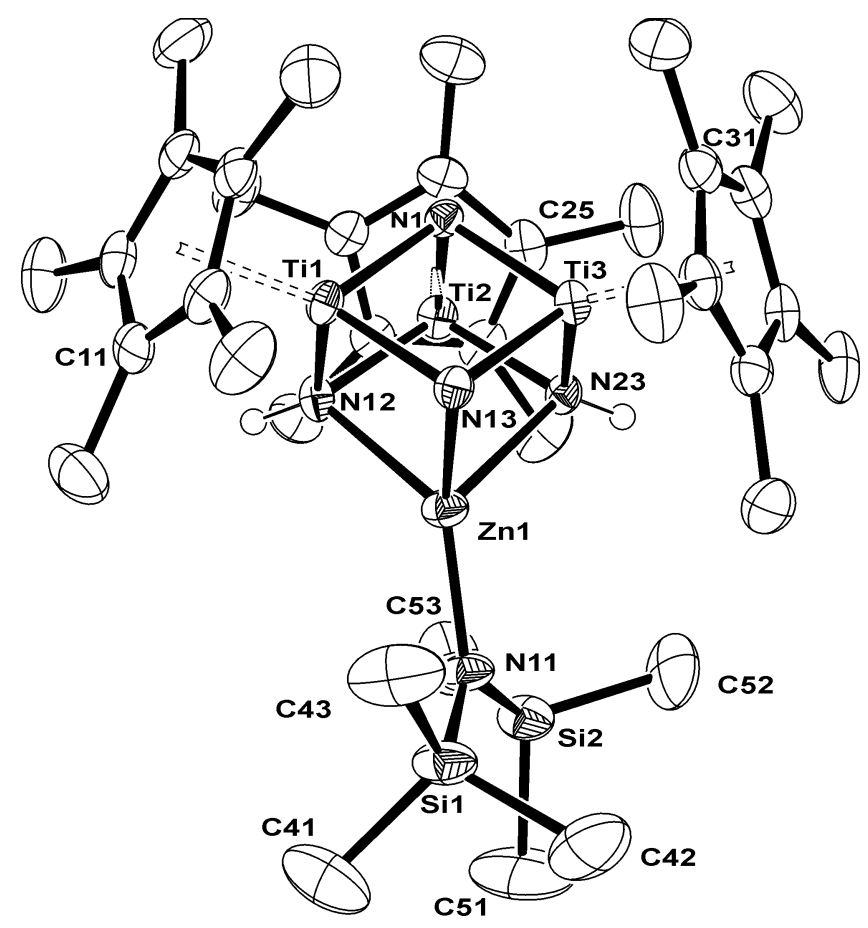

Figure 3. Perspective view of $\left[\left\{\left(\mathrm{Me}_{3} \mathrm{Si}\right)_{2} \mathrm{~N}\right\} \mathrm{Zn}\left\{\left(\mu_{3}-\mathrm{N}\right)\left(\mu_{3}-\mathrm{NH}\right)_{2} \mathrm{Ti}_{3}\left(\eta^{5}-\mathrm{C}_{5} \mathrm{Me}_{5}\right)_{3}\left(\mu_{3}-\mathrm{N}\right)\right\}\right]$ (9) with thermal ellipsoids at the 50\% probability level.

Table 4. Selected lengths $(\AA)$ and angles $\left(^{\circ}\right)$ for 9.

\begin{tabular}{lrlr}
\hline $\mathrm{Zn}(1)-\mathrm{N}(12)$ & $2.174(2)$ & $\mathrm{Zn}(1)-\mathrm{N}(13)$ & $2.132(2)$ \\
$\mathrm{Zn}(1)-\mathrm{N}(23)$ & $2.209(2)$ & $\mathrm{Zn}(1)-\mathrm{N}(11)$ & $1.916(2)$ \\
$\mathrm{N}(1)-\mathrm{Ti}(1)$ & $1.936(2)$ & $\mathrm{N}(1)-\mathrm{Ti}(3)$ & $1.939(2)$ \\
$\mathrm{N}(1)-\mathrm{Ti}(2)$ & $1.950(2)$ & $\mathrm{N}(11)-\mathrm{Si}(2)$ & $1.721(3)$ \\
$\mathrm{N}(11)-\mathrm{Si}(1)$ & $1.722(3)$ & $\mathrm{N}(12)-\mathrm{Ti}(1)$ & $1.961(2)$ \\
$\mathrm{N}(12)-\mathrm{Ti}(2)$ & $1.966(2)$ & $\mathrm{N}(13)-\mathrm{Ti}(1)$ & $1.957(2)$ \\
$\mathrm{N}(13)-\mathrm{Ti}(3)$ & $1.972(2)$ & $\mathrm{N}(23)-\mathrm{Ti}(2)$ & $1.923(2)$ \\
$\mathrm{N}(23)-\mathrm{Ti}(3)$ & $1.930(2)$ & $\mathrm{Ti} \cdot \mathrm{Ti} \mathrm{av}$ & $2.836(1)$ \\
$\mathrm{N}(12)-\mathrm{Zn}(1)-\mathrm{N}(13)$ & $87.1(1)$ & $\mathrm{N}(12)-\mathrm{Zn}(1)-\mathrm{N}(23)$ & $84.6(1)$ \\
$\mathrm{N}(13)-\mathrm{Zn}(1)-\mathrm{N}(23)$ & $86.2(1)$ & $\mathrm{N}(12)-\mathrm{Zn}(1)-\mathrm{N}(11)$ & $134.1(1)$ \\
$\mathrm{N}(13)-\mathrm{Zn}(1)-\mathrm{N}(11)$ & $126.8(1)$ & $\mathrm{N}(23)-\mathrm{Zn}(1)-\mathrm{N}(11)$ & $122.8(1)$ \\
$\mathrm{Ti}(1)-\mathrm{N}(1)-\mathrm{Ti}(3)$ & $94.3(1)$ & $\mathrm{Ti}(1)-\mathrm{N}(1)-\mathrm{Ti}(2)$ & $94.0(1)$ \\
$\mathrm{Ti}(3)-\mathrm{N}(1)-\mathrm{Ti}(2)$ & $93.2(1)$ & $\mathrm{Si}(2)-\mathrm{N}(11)-\mathrm{Si}(1)$ & $123.8(1)$ \\
$\mathrm{Si}(2)-\mathrm{N}(11)-\mathrm{Zn}(1)$ & $112.9(1)$ & $\mathrm{Si}(1)-\mathrm{N}(11)-\mathrm{Zn}(1)$ & $122.6(1)$ \\
$\mathrm{Ti}(1)-\mathrm{N}(12)-\mathrm{Ti}(2)$ & $92.7(1)$ & $\mathrm{Ti}(1)-\mathrm{N}(12)-\mathrm{Zn}(1)$ & $86.4(1)$ \\
$\mathrm{Ti}(2)-\mathrm{N}(12)-\mathrm{Zn}(1)$ & $88.3(1)$ & $\mathrm{Ti}(1)-\mathrm{N}(13)-\mathrm{Ti}(3)$ & $92.6(1)$ \\
$\mathrm{Ti}(1)-\mathrm{N}(13)-\mathrm{Zn}(1)$ & $87.7(1)$ & $\mathrm{Ti}(3)-\mathrm{N}(13)-\mathrm{Zn}(1)$ & $87.8(1)$ \\
$\mathrm{Ti}(2)-\mathrm{N}(23)-\mathrm{Ti}(3)$ & $94.4(1)$ & $\mathrm{Ti}(2)-\mathrm{N}(23)-\mathrm{Zn}(1)$ & $88.4(1)$ \\
$\mathrm{Ti}(3)-\mathrm{N}(23)-\mathrm{Zn}(1)$ & $86.7(1)$ & $\mathrm{N}(1)-\mathrm{Ti}(1)-\mathrm{N}(13)$ & $86.8(1)$ \\
$\mathrm{N}(1)-\mathrm{Ti}(1)-\mathrm{N}(12)$ & $86.9(1)$ & $\mathrm{N}(13)-\mathrm{Ti}(1)-\mathrm{N}(12)$ & $98.5(1)$ \\
$\mathrm{N}(23)-\mathrm{Ti}(2)-\mathrm{N}(1)$ & $86.1(1)$ & $\mathrm{N}(23)-\mathrm{Ti}(2)-\mathrm{N}(12)$ & $98.6(1)$ \\
$\mathrm{N}(1)-\mathrm{Ti}(2)-\mathrm{N}(12)$ & $86.3(1)$ & $\mathrm{N}(23)-\mathrm{Ti}(3)-\mathrm{N}(1)$ & $86.2(1)$ \\
$\mathrm{N}(23)-\mathrm{Ti}(3)-\mathrm{N}(13)$ & $98.9(1)$ & $\mathrm{N}(1)-\mathrm{Ti}(3)-\mathrm{N}(13)$ & $86.2(1)$ \\
\hline
\end{tabular}


The molecular structure of $\mathbf{9}$ confirms the azaheterometallocubane core $\left[\mathrm{ZnTi}_{3} \mathrm{~N}_{4}\right]$ of the complex and the tridentate coordination of the " $\left\{\left(\mu_{3}-\mathrm{N}\right)\left(\mu_{3}-\mathrm{NH}\right)_{2} \mathrm{Ti}_{3}\left(\eta^{5}-\mathrm{C}_{5} \mathrm{Me}_{5}\right)_{3}\left(\mu_{3}-\mathrm{N}\right)\right\}^{-}$" ligand to zinc. The geometry about the zinc center is best described as distorted tetrahedral, with intraligand $\mathrm{N}-\mathrm{Zn}-\mathrm{N}$ angles spanning 84.6(1)-87.1(1) ${ }^{\circ}$ and interligand $\mathrm{N}-\mathrm{Zn}-\mathrm{N}(11)$ angles in the range 122.8(1)-134.1(1) $)^{\circ}$. The trimethylsilyl groups of the amido ligand are oriented in an alternate disposition with respect to the pentamethylcyclopentadienyl rings of the preorganized triaza ligand to decrease the steric repulsion. The zinc-nitrogen bond lengths within the cube core are in the range 2.132(2)-2.209(2) $\AA$ and are longer than that found for the amido ligand [Zn-N(11) $1.916(2) \AA]$, in a similar way to the (guanidinato)(amido) complex $\left[\mathrm{Zn}(\mathrm{hpp})\left\{\mathrm{N}\left(\mathrm{SiMe}_{3}\right)_{2}\right\}\right]_{2}$ reported by Coles et al. ${ }^{42}$ The overall structure of $\mathbf{9}$ is very similar to that determined for the magnesium amido analogue $\left[\left\{\left(\mathrm{Me} e_{3} \mathrm{Si}\right)_{2} \mathrm{~N}\right\} \operatorname{Mg}\left\{\left(\mu_{3}-\mathrm{N}\right)\left(\mu_{3}-\mathrm{NH}\right)_{2} \mathrm{Ti}_{3}\left(\eta^{5}-\right.\right.\right.$ $\left.\left.\left.\mathrm{C}_{5} \mathrm{Me}_{5}\right)_{3}\left(\mu_{3}-\mathrm{N}\right)\right\}\right],{ }^{19}$ but showing slightly shorter $\mathrm{Zn}-\mathrm{N}$ bond lengths and wider $\mathrm{N}-\mathrm{Zn}-\mathrm{N}$ intraligand angles due to the smaller size of the zinc atom.

Alkyl complexes 5-8 are stable in benzene- $\mathrm{d}_{6}$ solutions at ambient light or temperatures about $100{ }^{\circ} \mathrm{C}$. In contrast, the magnesium analogous $\left[\mathrm{RMg}\left\{\left(\mu_{3}-\mathrm{N}\right)\left(\mu_{3}-\mathrm{NH}\right)_{2} \mathrm{Ti}_{3}\left(\eta^{5}-\mathrm{C}_{5} \mathrm{Me}_{5}\right)_{3}\left(\mu_{3^{-}}\right.\right.\right.$ N)\}] undergo Schlenk-type redistribution reactions at room temperature to give the cornershared nitrido complex $\left[\mathrm{Mg}\left\{\left(\mu_{3}-\mathrm{N}\right)\left(\mu_{3}-\mathrm{NH}\right)_{2} \mathrm{Ti}_{3}\left(\eta^{5}-\mathrm{C}_{5} \mathrm{Me}_{5}\right)_{3}\left(\mu_{3}-\mathrm{N}\right)\right\}_{2}\right] .{ }^{19} \quad$ Furthermore, compounds 5-8 did not react with one equivalent of $\mathbf{1}$ even at high temperatures. On the other hand, the bis(trimethylsilyl)amido derivative $\mathbf{9}$ is light sensitive in solution and decomposes, slowly at room temperature and rapidly at higher temperatures, to give the alkyl derivative $\left[\left\{\mathrm{Me}_{3} \mathrm{Si}(\mathrm{H}) \mathrm{N}(\mathrm{Me})_{2} \mathrm{SiCH}_{2}\right\} \mathrm{Zn}\left\{\left(\mu_{3}-\mathrm{N}\right)\left(\mu_{3}-\mathrm{NH}\right)_{2} \mathrm{Ti}_{3}\left(\eta^{5}-\mathrm{C}_{5} \mathrm{Me}_{5}\right)_{3}\left(\mu_{3}-\mathrm{N}\right)\right\}\right]$ (10) (Scheme 2). No intermediates or secondary products were identified in the NMR spectra by monitoring the reaction course of a benzene- $\mathrm{d}_{6}$ solution of $\mathbf{9}$ exposed to ambient light. Complex $\mathbf{1 0}$ was obtained as a brown solid in $79 \%$ yield by irradiation of toluene solutions of 9 with the light emitted by a sun lamp for 20 hours. ${ }^{1} \mathrm{H}$ and ${ }^{13} \mathrm{C}$ NMR spectra of $\mathbf{1 0}$ reveal resonances for two 
different $\eta^{5}-\mathrm{C}_{5} \mathrm{Me}_{5}$ ligands in a 2:1 ratio and are consistent with a $\mathrm{C}_{\mathrm{s}}$ symmetry in solution. The methylene $\mathrm{CH}_{2}$ group of $\mathbf{1 0}$ shows a singlet resonance at $-0.69 \mathrm{ppm}$ in the ${ }^{1} \mathrm{H}$ NMR spectrum and a triplet resonance $\left({ }^{1} \mathrm{~J}_{\mathrm{C}-\mathrm{H}}=115 \mathrm{~Hz}\right)$ at $-11.0 \mathrm{ppm}$ in the ${ }^{13} \mathrm{C}$ NMR spectrum. These chemical shifts are similar to those found for the methylene group in the spectra of $\left[\left(\mathrm{Me}_{3} \mathrm{SiCH}_{2}\right) \mathrm{Zn}\left\{\left(\mu_{3}-\mathrm{N}\right)\left(\mu_{3}-\mathrm{NH}\right)_{2} \mathrm{Ti}_{3}\left(\eta^{5}-\mathrm{C}_{5} \mathrm{Me}_{5}\right)_{3}\left(\mu_{3}-\mathrm{N}\right)\right\}\right](\mathbf{5}),-0.77\left(\mathrm{CH}_{2}\right)$ and $-13.3\left(\mathrm{CH}_{2}\right)$ ppm respectively. However, the ${ }^{1} \mathrm{H}$ NMR spectrum of $\mathbf{1 0}$ did not allow the unambiguous identification of the $\mathrm{NH}$ resonance for the $\mathrm{CH}_{2} \mathrm{Si}(\mathrm{Me})_{2} \mathrm{~N}(\mathrm{H}) \mathrm{SiMe}_{3}$ group.

In order to test other ways to prepare the azaheterometallocubane complexes of zinc, we tried reactions of the dichloride complex $\left[\mathrm{Cl}_{2} \mathrm{Zn}\left\{\left(\mu_{3}-\mathrm{NH}\right)_{3} \mathrm{Ti}_{3}\left(\eta^{5}-\mathrm{C}_{5} \mathrm{Me}_{5}\right)_{3}\left(\mu_{3}-\mathrm{N}\right)\right\}\right]$ (2) with several organolithium reagents (Scheme 3). Thus, treatment of 2 with 2 equiv of lithium (trimethylsilyl)methyl, (trimethylsilyl)cyclopentadienyl or bis(trimethylsilyl)amido derivatives in toluene at room temperature afforded the alkyl or amido zinc complexes $\left[\operatorname{RZn}\left\{\left(\mu_{3}-\mathrm{N}\right)\left(\mu_{3}-\right.\right.\right.$ $\left.\left.\mathrm{NH})_{2} \mathrm{Ti}_{3}\left(\eta^{5}-\mathrm{C}_{5} \mathrm{Me}_{5}\right)_{3}\left(\mu_{3}-\mathrm{N}\right)\right\}\right]\left[\mathrm{R}=\mathrm{CH}_{2} \mathrm{SiMe}_{3}(\mathbf{5}), \mathrm{C}_{5} \mathrm{H}_{4} \mathrm{SiMe}_{3}(\mathbf{8}), \mathrm{N}\left(\mathrm{SiMe}_{3}\right)_{2}(\mathbf{9})\right]$ in good yields (68-78\%). Analogous reactions of 2 with two equivalents of lithium cyclopentadienyl $\left[\mathrm{Li}\left(\mathrm{C}_{5} \mathrm{H}_{5}\right)\right]$ or lithium indenyl $\left[\mathrm{Li}\left(\mathrm{C}_{9} \mathrm{H}_{7}\right)\right]$ reagents at room temperature gave the new cyclopentadienyl or indenyl zinc derivatives $\left[R Z n\left\{\left(\mu_{3}-\mathrm{N}\right)\left(\mu_{3}-\mathrm{NH}\right)_{2} \mathrm{Ti}_{3}\left(\eta^{5}-\mathrm{C}_{5} \mathrm{Me}_{5}\right)_{3}\left(\mu_{3}-\mathrm{N}\right)\right\}\right][\mathrm{R}$ $\left.=\mathrm{C}_{5} \mathrm{H}_{5}(\mathbf{1 1}), \mathrm{C}_{9} \mathrm{H}_{7}(\mathbf{1 2})\right]$ as red solids in good yields $(72-73 \%)$.

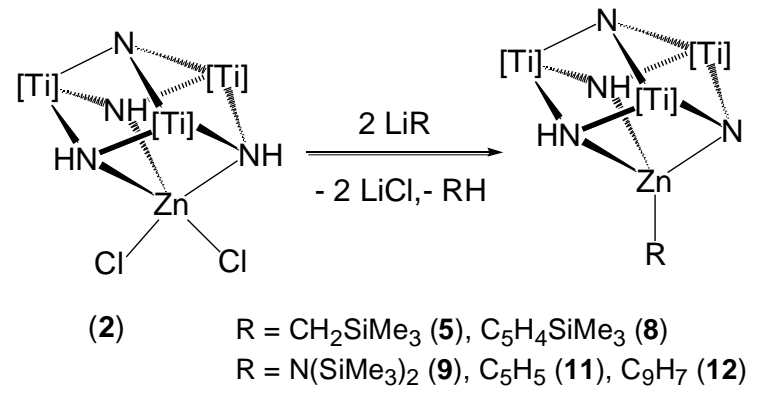

Scheme 3. Reactions of 2 with LiR. [Ti] $=\operatorname{Ti}\left(\eta^{5}-\mathrm{C}_{5} \mathrm{Me}_{5}\right)$

Complexes 11 and 12 exhibit a poor solubility in hexane but are very soluble in toluene or benzene, and the solutions are stable under argon atmosphere at ambient light and 
temperature conditions. Compounds $\mathbf{1 1}$ and $\mathbf{1 2}$ were characterized by spectral and analytical methods. Mass spectra (EI, $70 \mathrm{eV}$ ) show the expected molecular peaks suggesting a singlecube aggregation for the complexes in the gas phase. ${ }^{1} \mathrm{H}$ and ${ }^{13} \mathrm{C}\left\{{ }^{1} \mathrm{H}\right\}$ NMR spectra in benzene- $\mathrm{d}_{6}$ at room temperature show resonance signals for two $\eta^{5}-\mathrm{C}_{5} \mathrm{Me}_{5}$ groups in a 2:1 ratio, one cyclopentadienyl or indenyl ligand and a broad signal for the NH groups. In order to investigate the coordination mode of the cyclopentadienyl or indenyl ligands to the zinc center, we studied a toluene- $\mathrm{d}_{8}$ solution of complex $\mathbf{1 2}$ by low temperature ${ }^{1} \mathrm{H}$ NMR spectroscopy in a $500 \mathrm{MHz}$ spectrometer. The room temperature ${ }^{1} \mathrm{H}$ NMR spectrum of 12 showed the following resonances for the indenyl group: one $\operatorname{doublet}\left({ }^{3} \mathbf{J}=3.0 \mathrm{~Hz}\right)$ at $\delta 5.53$ for the $\mathrm{H}_{1}$ and $\mathrm{H}_{3}$, one triplet at $\delta 6.98$ for the $\mathrm{H}_{2}$ and a sharp $\mathrm{AA}^{\prime} \mathrm{BB}^{\prime}$ pattern at $\delta 7.59-7.23$ for the $\mathrm{H}_{4}, \mathrm{H}_{5}, \mathrm{H}_{6}$ and $\mathrm{H}_{7}$. Upon cooling at $-20^{\circ} \mathrm{C}$ the resonance signal at $\delta 5.53$ began to broaden and at $-94{ }^{\circ} \mathrm{C}$ gave two very broad resonances centered at approximately $\delta 4.6$ and 7.0 which coalesced at about $-89^{\circ} \mathrm{C}$. This nonequivalence of the protons of the indenyl ligand at low temperature is consistent with a $\eta^{1}(\sigma)$ coordination of the indenyl ligand by the $\mathrm{C}_{1}$ or $\mathrm{C}_{3}$ to the zinc center. The fluxional process observed can be explained by an intramolecular 1,3 migration of the zinc on the indenyl ligand that is very fast on the NMR time scale at room temperature and slower at $-94^{\circ} \mathrm{C}$ (Scheme 4$)$.

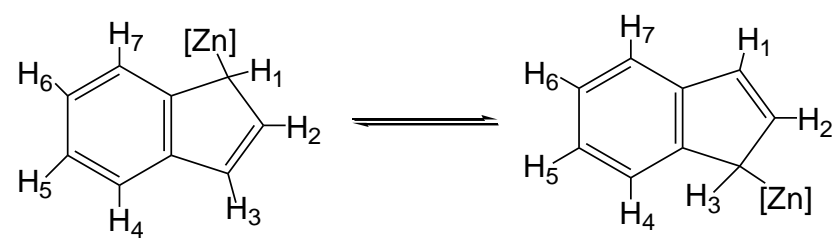

Scheme. 4. 1,3 Migration of the [Zn] fragment on the indenyl ligand in $\mathbf{1 2}$.

An analogous 1,3 migration has been investigated in detail by Casey on several indenylrhenium derivatives. ${ }^{43}$ In those studies on the fluxional processes, and in many others previously, ${ }^{44}$ the authors observed a smaller barrier for $\eta^{1}$-cyclopentadienyl systems compared with the $\eta^{1}$-indenyl systems. Therefore, we did not study the fluxional process occurring on 
complex 11, but the NMR spectra are consistent with $\eta^{1}(\sigma)$ coordination of the cyclopentadienyl ligand bonded to zinc and a more rapid 1,2 migration of the zinc on the cyclopentadienyl ring.

Azaheterometallocubanes of copper(I). Treatment of $\left[\left\{\mathrm{Ti}\left(\eta^{5}-\mathrm{C}_{5} \mathrm{Me}_{5}\right)(\mu-\mathrm{NH})\right\}_{3}\left(\mu_{3}-\mathrm{N}\right)\right]$ (1) with anhydrous copper(I) halides (1 equiv) in toluene at room temperature affords the adducts $\left[\mathrm{XCu}\left\{\left(\mu_{3}-\mathrm{NH}\right)_{3} \mathrm{Ti}_{3}\left(\eta^{5}-\mathrm{C}_{5} \mathrm{Me}_{5}\right)_{3}\left(\mu_{3}-\mathrm{N}\right)\right\}\right](\mathrm{X}=\mathrm{Cl}(\mathbf{1 3}), \mathrm{I}(\mathbf{1 4}))$ in good yields $(72$ and 65\%, respectively) (Scheme 5). Complexes 13 and 14 were obtained as red solids, which are very soluble in toluene and scarcely in hexane. Mass spectrum (EI, $70 \mathrm{eV}$ ) of $\mathbf{1 3}$ shows the molecular peak for a single cube structure whereas the spectrum of $\mathbf{1 4}$ reveals the ion $[\mathrm{M}-\mathrm{I}]^{+}$ as that of the higher relation mass/charge. ${ }^{1} \mathrm{H}$ and ${ }^{13} \mathrm{C}\left\{{ }^{1} \mathrm{H}\right\}$ NMR spectra of $\mathbf{1 3}$ and 14 in benzene- $\mathrm{d}_{6}$ reveal resonances for equivalent $\mathrm{NH}$ and $\eta^{5}-\mathrm{C}_{5} \mathrm{Me}_{5}$ groups. The $\mathrm{NH}$ resonance signals $(\delta=11.70$ and 11.68) are shifted to higher field than that found for $\mathbf{1}(\delta=13.40)$, whereas the resonance signal for the $C_{5} \mathrm{Me}_{5}$ groups $(\delta=119.4$ and 119.5) are shifted downfield with respect to that found for $\mathbf{1}(\delta=117.1)$. These NMR data are similar to those described above for $\mathbf{2}$ and are consistent with a tridentate coordination of $\mathbf{1}$ to the copper center and a $\mathrm{C}_{3 \mathrm{v}}$ symmetry in solution.

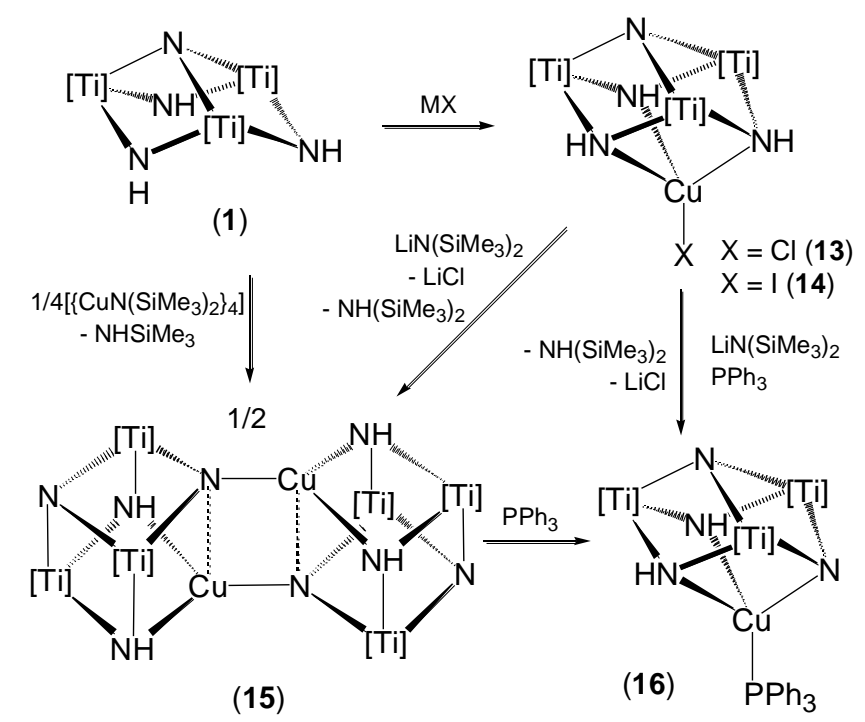

Scheme 5. Synthesis of copper(I) azaheterometallocubane complexes. [Ti] $=\mathrm{Ti}\left(\eta^{5}-\mathrm{C}_{5} \mathrm{Me}_{5}\right)$ 
Treatment of the chloride derivative $\mathbf{1 3}$ with lithium bis(trimethylsilyl)amido in toluene at room temperature leads to bis(trimethylsilyl)amine and the precipitation of huge red crystals of the dicubane complex $\left[\left\{\mathrm{Cu}\left(\mu_{4}-\mathrm{N}\right)\left(\mu_{3}-\mathrm{NH}\right)_{2} \mathrm{Ti}_{3}\left(\eta^{5}-\mathrm{C}_{5} \mathrm{Me}_{5}\right)_{3}\left(\mu_{3}-\mathrm{N}\right)\right\}_{2}\right](\mathbf{1 5})$ together with a fine powder of $\mathrm{LiCl}$. Lithium chloride can be removed from the mixture after rinsing with toluene for several times to give $\mathbf{1 5}$ in $51 \%$ yield. Complex $\mathbf{1 5}$ can be obtained in higher yield (73\%) through the reaction of 1 with $\left[\left\{\mathrm{CuN}\left(\mathrm{SiMe}_{3}\right)_{2}\right\}_{4}\right]^{31,32}\left(0.25\right.$ equiv) in toluene at $150{ }^{\circ} \mathrm{C}$ for 6 days. A mixture of 1 with $\left[\left\{\mathrm{CuN}\left(\mathrm{SiMe}_{3}\right)_{2}\right\}_{4}\right]$ in benzene- $\mathrm{d}_{6}$ was monitored by NMR spectroscopy at different temperatures. After heating at $40{ }^{\circ} \mathrm{C}$, red crystals precipitated at the bottom of the tube and resonances due to bis(trimethylsilyl)amine were detected in the spectra. However, the reaction rate was very slow at temperatures below $120{ }^{\circ} \mathrm{C}$, probably due to the high stability of the tetranuclear structure determined for the copper amido reagent. ${ }^{32,45}$ Complex 15 was characterized by IR spectroscopy and C,H,N microanalysis, as well as by an X-ray crystal structure determination for suitable crystals of $15 \cdot \mathrm{C}_{6} \mathrm{D}_{6}$ grown in the treatment of 13 with $\mathrm{LiN}\left(\mathrm{SiMe}_{3}\right)_{2}$ in benzene-d 6 without any stirring.

The X-ray structure of $\mathbf{1 5}$ is presented in Figure 4 while selected bond and angles are shown in Table 5. Crystals of $\mathbf{1 5}$ bear one benzene solvent molecule per double cube complex. The molecule lies on an inversion center and shows an edge-linked double cube arrangement with two cube type $\left[\mathrm{CuTi}_{3} \mathrm{~N}_{4}\right]$ units, resembling the molybdenum-copper sulfido complex published by Shibahara and coworkers. ${ }^{46}$ The two $\left\{\mathrm{Cu}\left(\mu_{3}-\mathrm{N}\right)\left(\mu_{3}-\mathrm{NH}\right)_{2} \mathrm{Ti}_{3}\left(\eta^{5}-\mathrm{C}_{5} \mathrm{Me}_{5}\right)_{3}\left(\mu_{3}-\mathrm{N}\right)\right\}$ moieties adopt a mutually staggered disposition which minimizes the steric repulsion between the bulky pentamethylcyclopentadienyl ligands, in a similar way to the previously reported edge-linked Group 1 azaheterometallocubane complexes. ${ }^{17}$ 


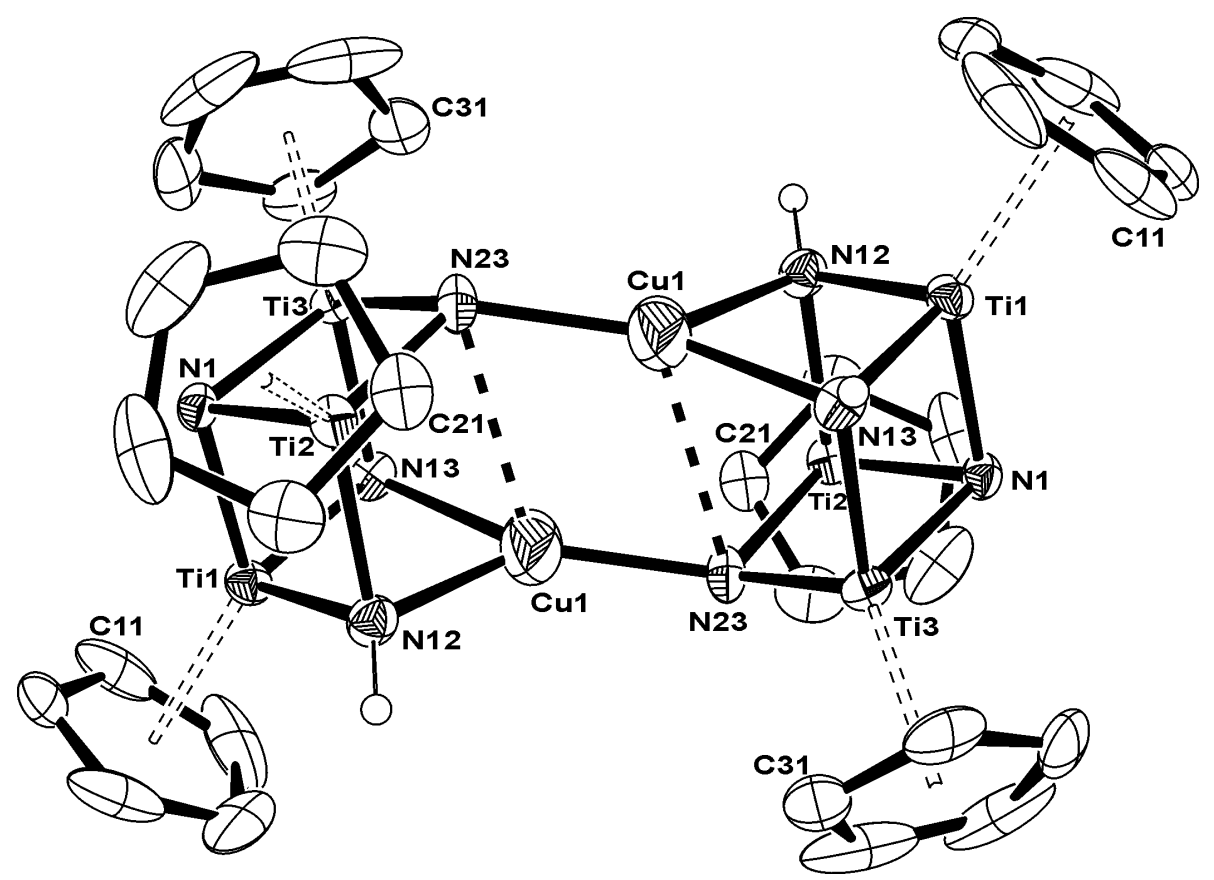

Figure 4. Perspective view of $\left[\left\{\mathrm{Cu}\left(\mu_{4}-\mathrm{N}\right)\left(\mu_{3}-\mathrm{NH}\right)_{2} \mathrm{Ti}_{3}\left(\eta^{5}-\mathrm{C}_{5} \mathrm{Me}_{5}\right)_{3}\left(\mu_{3}-\mathrm{N}\right)\right\}_{2}\right] \quad$ (15) with thermal ellipsoids at the 50\% probability level. The benzene solvent molecule and methyl groups of the pentamethylcyclopentadienyl rings are omitted for clarity.

Table 5. Selected lengths $(\AA)$ and angles $\left(^{\circ}\right)$ for 15.

\begin{tabular}{lrlr}
\hline $\mathrm{Cu}(1)-\mathrm{N}(12)$ & $2.218(5)$ & $\mathrm{Cu}(1)-\mathrm{N}(13)$ & $2.101(5)$ \\
$\mathrm{Cu}(1)-\mathrm{N}(23)^{\mathrm{a}}$ & $1.912(5)$ & $\mathrm{Cu}(1) \cdots \mathrm{N}(23)$ & $2.690(5)$ \\
$\mathrm{Cu}(1) \cdots \mathrm{Cu}(1)$ & $2.614(2)$ & $\mathrm{N}(1)-\mathrm{Ti}(1)$ & $1.916(4)$ \\
$\mathrm{N}(1)-\mathrm{Ti}(3)$ & $1.947(4)$ & $\mathrm{N}(1)-\mathrm{Ti}(2)$ & $1.952(4)$ \\
$\mathrm{N}(12)-\mathrm{Ti}(1)$ & $1.968(5)$ & $\mathrm{N}(12)-\mathrm{Ti}(2)$ & $2.007(5)$ \\
$\mathrm{N}(13)-\mathrm{Ti}(1)$ & $1.975(5)$ & $\mathrm{N}(13)-\mathrm{Ti}(3)$ & $1.987(5)$ \\
$\mathrm{N}(23)-\mathrm{Ti}(2)$ & $1.882(5)$ & $\mathrm{N}(23)-\mathrm{Ti}(3)$ & $1.891(5)$ \\
$\mathrm{N}(13)-\mathrm{Cu}(1)-\mathrm{N}(12)$ & $89.4(2)$ & $\mathrm{N}(23)^{\mathrm{a}}-\mathrm{Cu}(1)-\mathrm{N}(13)$ & $140.2(2)$ \\
$\mathrm{N}(23)^{\mathrm{a}}-\mathrm{Cu}(1)-\mathrm{N}(12)$ & $130.0(2)$ & $\mathrm{N}(23)^{\mathrm{a}}-\mathrm{Cu}(1)-\mathrm{N}(23)$ & $113.2(2)$ \\
$\mathrm{Ti}(1)-\mathrm{N}(1)-\mathrm{Ti}(2)$ & $95.4(2)$ & $\mathrm{Ti}(1)-\mathrm{N}(1)-\mathrm{Ti}(3)$ & $95.2(2)$ \\
$\mathrm{Ti}(2)-\mathrm{N}(1)-\mathrm{Ti}(3)$ & $90.8(2)$ & $\mathrm{Ti}(1)-\mathrm{N}(12)-\mathrm{Ti}(2)$ & $92.0(2)$ \\
$\mathrm{Ti}(1)-\mathrm{N}(12)-\mathrm{Cu}(1)$ & $81.8(2)$ & $\mathrm{Ti}(2)-\mathrm{N}(12)-\mathrm{Cu}(1)$ & $95.5(2)$ \\
$\mathrm{Ti}(1)-\mathrm{N}(13)-\mathrm{Ti}(3)$ & $92.1(2)$ & $\mathrm{Ti}(1)-\mathrm{N}(13)-\mathrm{Cu}(1)$ & $84.7(2)$ \\
$\mathrm{Ti}(3)-\mathrm{N}(13)-\mathrm{Cu}(1)$ & $97.1(2)$ & $\mathrm{Ti}(2)-\mathrm{N}(23)-\mathrm{Ti}(3)$ & $94.8(2)$ \\
$\mathrm{Ti}(2)-\mathrm{N}(23)-\mathrm{Cu}(1)^{\mathrm{a}}$ & $121.0(3)$ & $\mathrm{Ti}(3)-\mathrm{N}(23)-\mathrm{Cu}(1)^{\mathrm{a}}$ & $127.8(3)$ \\
$\mathrm{N}(1)-\mathrm{Ti}(1)-\mathrm{N}(12)$ & $87.1(2)$ & $\mathrm{N}(1)-\mathrm{Ti}(1)-\mathrm{N}(13)$ & $86.8(2)$ \\
$\mathrm{N}(12)-\mathrm{Ti}(1)-\mathrm{N}(13)$ & $100.8(2)$ & $\mathrm{N}(1)-\mathrm{Ti}(2)-\mathrm{N}(23)$ & $87.1(2)$ \\
$\mathrm{N}(1)-\mathrm{Ti}(2)-\mathrm{N}(12)$ & $85.0(2)$ & $\mathrm{N}(23)-\mathrm{Ti}(2)-\mathrm{N}(12)$ & $103.6(2)$ \\
$\mathrm{N}(1)-\mathrm{Ti}(3)-\mathrm{N}(23)$ & $87.0(2)$ & $\mathrm{N}(1)-\mathrm{Ti}(3)-\mathrm{N}(13)$ & $85.6(2)$ \\
$\mathrm{N}(23)-\mathrm{Ti}(3)-\mathrm{N}(13)$ & $103.1(2)$ & & \\
\hline
\end{tabular}

${ }^{\mathrm{a}}$ Intercube atoms 
Each copper atom in $\mathbf{1 5}$ presents an almost trigonal environment (sum of angles $359.6^{\circ}$ ) with $\mathrm{Cu}-\mathrm{N}$ bond distances from $1.912(5)$ to $2.218(5) \AA$ A. These lengths are in the same range than those found for other nitrogen polydentate ligands joined to copper ${ }^{47}$ and slightly longer than those reported for several tris(pyrazolyl)hydroboratecopper $(\mathrm{I})\left[\left\{\left(\mathrm{Tp}^{\mathrm{x}}\right) \mathrm{Cu}\right\}_{2}\right]$ derivatives. ${ }^{48}$ On the other hand, a triangular pyramidal environment could be proposed if the long range interaction of copper with the intracube $\mathrm{N}(23)$ atom $(2.690(5) \AA)$ is taken into consideration. Bond distances and angles involving titanium and nitrogen atoms of the same cube do not differ significantly with respect to the preorganized organometallic ligand $1,{ }^{8}$ with Ti-N 1.947(5) $\AA$ average [1.924(6) $\AA$ in 1], Ti-N-Ti 93.4(2) ${ }^{\circ}$ average [93.7(3) ${ }^{\circ}$ in 1], N1-Ti-N $87.0(2)^{\circ}$ average $\left[85.9(3)^{\circ}\right.$ in 1 ] and only 5 degrees narrower N-Ti-N angles, $102.5(2)^{\circ}$ average in $\mathbf{1 5}$ and $107.5(3)^{\circ}$ in $\mathbf{1}$. This latter deviation can be attributed to the coordination of the nitrogen atoms to copper.

Crystals of $\mathbf{1 5}$ are not soluble in common organic solvents (benzene- $\mathrm{d}_{6}$, chloroform- $\mathrm{d}_{1}$, pyridine- $\mathrm{d}_{5}, \ldots$ ) precluding its characterization by NMR spectroscopy. However, treatment of 15 with triphenylphosphane ( 2 equiv) in benzene- $\mathrm{d}_{6}$ at $80{ }^{\circ} \mathrm{C}$ gave an orange solution where the single-cube complex $\left[\left(\mathrm{Ph}_{3} \mathrm{P}\right) \mathrm{Cu}\left\{\left(\mu_{3}-\mathrm{N}\right)\left(\mu_{3}-\mathrm{NH}\right)_{2} \mathrm{Ti}_{3}\left(\eta^{5}-\mathrm{C}_{5} \mathrm{Me}_{5}\right)_{3}\left(\mu_{3}-\mathrm{N}\right)\right\}\right] \quad$ (16) was characterized by NMR spectroscopy. Complex 16 was prepared in good yield (87\%) by the direct reaction of a toluene solution of $\mathbf{1 3}$ and triphenylphospane with [ $\left.\mathrm{LiN}\left(\mathrm{SiMe}_{3}\right)_{2}\right]$.

Compound 16 was obtained as an orange solid, which is very soluble in toluene and hexane, and was characterized by spectral and analytical techniques. ${ }^{1} \mathrm{H}$ and ${ }^{13} \mathrm{C}\left\{{ }^{1} \mathrm{H}\right\}$ NMR spectra in benzene- $\mathrm{d}_{6}$ at room temperature show resonance signals for two $\eta^{5}-\mathrm{C}_{5} \mathrm{Me}_{5}$ groups in a 2:1 ratio, one triphenylphospane ligand and a broad signal for the $\mathrm{NH}$ groups. These data are consistent with a $\mathrm{C}_{\mathrm{s}}$ symmetry and a single cube structure in solution. The tetrahedral environment around the copper center in $\mathbf{1 6}$ would be similar to those reported for several $\mathrm{Tp}^{\mathrm{x}} \mathrm{Cu}\left(\mathrm{PPh}_{3}\right)$ derivatives. ${ }^{49}$ 


\section{Conclusion}

In summary, the imido-nitrido titanium complex $\left[\left\{\mathrm{Ti}\left(\eta^{5}-\mathrm{C}_{5} \mathrm{Me}_{5}\right)(\mu-\mathrm{NH})\right\}_{3}\left(\mu_{3}-\mathrm{N}\right)\right](\mathbf{1})$ is capable of acting as a neutral ligand to give stable adducts with zinc(II) and copper(I) halides, while analogous derivatives with zinc and copper alkyl or amido groups undergo alkane or amine elimination to give complexes bearing the monoanionic $\left[\left\{\left(\mu_{3}-\mathrm{N}\right)\left(\mu_{3}-\mathrm{NH}\right)_{2} \mathrm{Ti}_{3}\left(\eta^{5}-\right.\right.\right.$ $\left.\left.\left.\mathrm{C}_{5} \mathrm{Me}_{5}\right)_{3}\left(\mu_{3}-\mathrm{N}\right)\right\}\right]^{-}$ligand. The coordination modes of $\mathbf{1}$ and its monoanionic form in those complexes resemble other well-established "non-organometallic" nitrogen-based ligands. The new heterometallic nitrido compounds here reported exhibit single-cube structures with $\left[\mathrm{MTi}_{3} \mathrm{~N}_{4}\right]$ central cores, excepting the copper derivative $\left[\left\{\mathrm{Cu}\left(\mu_{3}-\mathrm{N}\right)\left(\mu_{3}-\mathrm{NH}\right)_{2} \mathrm{Ti}_{3}\left(\eta^{5}-\right.\right.\right.$ $\left.\left.\left.\mathrm{C}_{5} \mathrm{Me}_{5}\right)_{3}\left(\mu_{3}-\mathrm{N}\right)\right\}_{2}\right]$ that shows a double cube structure with a $\left[\mathrm{Cu}_{2} \mathrm{Ti}_{6} \mathrm{~N}_{8}\right]$ core.

Acknowledgments. We are grateful to the Spanish MCYT (BQU2001-1499), DGICAM (GR/MAT/0621/2004), and the Universidad de Alcalá (CAM-UAH2005/062) for support of this research.

Supporting Information Available: X-ray crystallographic files in CIF format for complexes 3, 8,9 and 15. This material is available free of charge via the Internet at http://pubs.acs.org. 


\section{References}

(1) For general references, see: (a) Trofimenko, S. Scorpionates: The Coordination Chemistry of Polypyrazolylborate Ligands, Imperial College, 1999. (b) Pettinari, C.; Santini, C. in Comprehensive Coordination Chemistry II Vol. 1 (Eds.: McCleverty, J. A.; Meyer, T. J.), Elsevier, 2004, pp. 159-210. (c) Trofimenko, S. Chem Rev. 1993, 93, 943-980.

(2) For recent reviews, see: (a) Bigmore, H. R.; Lawrence, S. C.; Mountford, P.; Tredget, C. S. Dalton Trans. 2005, 635-651. (b) Pettinari, C.; Pettinari, R. Coord. Chem. Rev. 2005, 249, 525-543.

(3) For reviews, see: (a) Chaudhuri, P.; Wieghardt, K. Prog. Inorg. Chem. 1987, 35, 329-436. (b) Schmidt, J. A. R.; Giesbrecht, G. R.; Cui, C.; Arnold, J. Chem. Commun. 2003, 1025-1033.

(4) For a selection of 1,3,5-triazacyclohexane complexes of copper and zinc, see: (a) Fuchs, R.; Klüfers, P. J. Organomet. Chem. 1992, 424, 353-370. (b) Köhn, R. D.; Seifert, G.; KociokKöhn, G. Chem. Ber. 1996, 129, 1327-1333. (c) Haufe, M.; Köhn, R. D.; Weimann, R.; Seifert, G.; Zeigan, D. J. Organomet. Chem. 1996, 520, 121-129. (d) Haufe, M.; Köhn, R. D.; Kociok-Köhn, G.; Filippou, A. C. Inorg. Chem. Commun. 1998, 1, 263-266. (e) Köhn, R. D.; Seifert, G.; Pan, Z.; Mahon, M. F.; Kociok-Köhn, G. Angew. Chem., Int. Ed. 2003, 42, 793796. (f) Köhn, R. D.; Pan, Z.; Mahon, M. F.; Kociok-Köhn, G. Chem Commun. 2003, 12721273. (g) Köhn, R. D.; Pan, Z.; Mahon, M. F.; Kociok-Köhn, G. Dalton Trans. 2003, 22692275. (h) Köhn, R. D.; Pan, Z.; Haufe, M.; Kociok-Köhn, G. Dalton Trans. 2005, 2793-2797.

(5) For recent reviews, see: (a) Gade, L. H. Acc. Chem. Res. 2002, 35, 575-582. (b) Gade, L. H. J. Organomet. Chem. 2002, 661, 85-94.

(6) Tellers, D. M.; Skoog, S. J.; Bergman, R. G.; Gunnoe, T. B.; Harman, W. D. Organometallics 2000, 19, 2428-2432.

(7) (a) Kitajima, N.; Tolman, W. B. Prog. Inorg. Chem. 1995, 43, 419-513. (b) Sandford, M. S.; Henling, L. M.; Grubbs, R. H. Organometallics 1998, 17, 5384-5389, and references therein. 
(8) Roesky, H. W.; Bai, Y.; Noltemeyer, M. Angew. Chem. Int. Ed. Engl. 1989, 28, 754-755.

(9) Abarca, A.; Gómez-Sal, P.; Martín, A.; Mena, M.; Poblet, J. M.; Yélamos, C. Inorg. Chem. 2000, 39, 642-651.

(10) For general references of metal nitrido complexes, see: (a) Dehnicke, K.; Strähle, J. Angew. Chem. Int. Ed. Engl. 1981, 20, 413-426. (b) Nugent, W. A.; Mayer, J. M. Metal-Ligand Multiple Bonds, Wiley, New York, 1988. (c) Dehnicke, K.; Strähle, J. Angew. Chem. Int. Ed. Engl. 1992, 31, 955-978. (d) Dehnicke, K.; Weller, F.; Strähle J. Chem. Soc. Rev. 2001, 30, $125-135$

(11) Abarca, A.; Galakhov, M. V.; Gracia, J.; Martín, A.; Mena, M.; Poblet, J. M.; Sarasa, J. P.; Yélamos, C. Chem. Eur. J. 2003, 9, 2337-2346, and references therein concerning early transition metal nitrido complexes.

(12) Haack, K. J.; Goddard, R.; Pörschke, K. R. J. Am. Chem. Soc. 1997, 119, 7992-7999.

(13) (a) Oyama, S. T. (Ed.) The Chemistry of Transition Metal Carbides and Nitrides, Blackie Academic \& Professional, London, 1996. (b) Kniep, R. Pure Appl. Chem. 1997, 69, 185-191. (c) Niewa, R.; DiSalvo, F. J. Chem. Mater. 1998, 10, 2733-2752. (d) Gregory, D. H. J. Chem. Soc., Dalton Trans. 1999, 259-270.

(14) (a) Abarca, A.; Galakhov, M.; Gómez-Sal, P.; Martín, A.; Mena, M.; Poblet, J. M.; Santamaría, C.; Sarasa, J. P. Angew. Chem. Int. Ed. 2000, 39, 534-537. (b) Abarca, A.; Martín, A.; Mena, M.; Yélamos, C. Angew. Chem. Int. Ed. 2000, 39, 3460-3463.

(15) Freitag, K.; Gracia, J.; Martín, A.; Mena, M.; Poblet, J. M.; Sarasa, J. P.; Yélamos, C. Chem. Eur. J. 2001, 7, 3644-3651.

(16) (a) García-Castro, M.; Martín, A.; Mena, M.; Yélamos, C. Organometallics 2004, 23, 14961500. (b) García-Castro, M.; Gracia, J; Martín, A.; Mena, M.; Poblet. J. M.; Sarasa, J. P.; Yélamos, C. Chem. Eur. J. 2005, 11, 1030-1041. 
(17) García-Castro, M.; Martín, A.; Mena, M.; Pérez-Redondo, A.; Yélamos, C. Chem. Eur. J. 2001, 7, 647-651.

(18) Martín, A.; Mena, M.; Pérez-Redondo, A.; Yélamos, C. Inorg. Chem. 2004, 43, 2491-2498.

(19) Martín, A.; Mena, M.; Pérez-Redondo, A.; Yélamos, C. Organometallics 2002, 21, 33083310.

(20) (a) Reger, D. L. Comments Inorg. Chem. 1999, 21, 1-28. (b) Pettinari, C.; Cingolani, A.; Lobbia, G. G.; Marchetti, F.; Martini, D.; Pellei, M.; Pettinari, R.; Santini, C. Polyhedron, 2004, 23, 451-469.

(21) Westerhausen, M.; Rademacher, B.; Poll, W. J. Organomet. Chem. 1991, 421, 175-188.

(22) Schrock, R. R. J. Organomet. Chem. 1976, 122, 209-225.

(23) Bürger, H.; Sawodny, W.; Wannagat, U. J. Organomet. Chem. 1965, 3, 113-120.

(24) Bochmann, M.; Bwembya, G.; Webb, K. J. Inorg. Synth. 1997, 31, $19-24$.

(25) (a) Blom, A.; Boersma, J.; Budzelaar, P. H.; Fischer, B.; Haaland, A.; Volden, H. V.; Weidlein, J. Acta Chem. Scand. 1986, A40, 113-120. (b) Fischer, B.; Boersma, J.; van Koten, G.; Spek, A. L. New J. Chem. 1988, 12, 613-620.

(26) Budzelaar, P. H. M.; Boersma, J.; van der Kerk, G. J. M.; Spek, A. L.; Duisenberg, A. J. M. J. Organomet. Chem. 1985, 281, 123-130.

(27) Tessier-Youngs, C.; Beachley, O. T. Jr. Inorg. Synth. 1986, 24, 95-97.

(28) Jutzi, P.; Sauer, R. J. Organomet. Chem. 1973, 50, C29-C30.

(29) Dinnebier, R. E.; Behrens, U.; Olbrich, F. Organometallics 1997, 16, 3855-3858.

(30) Christopher, J. N.; Diamond, G. M.; Jordan, R. F.; Petersen, J. L. Organometallics 1996, 15, 4038-4044.

(31) Bürger, H.; Wannagat, U. Monatsh. Chem. 1964, 95, 1099-1102.

(32) James, A. M.; Laxman, R. K.; Fronczek, F. R., Maverick, A. W. Inorg. Chem. 1998, 37, 37853791. 
(33) Farrugia, L. J. J. App. Cryst. 1999, 32, 837-838.

(34) Sheldrick, G. M. SHELX97, Program for Crystal Structure Analysis (Release 97-2). Universität Göttingen, Germany, 1998.

(35) Block, B. P.; Florentine, R. A.; Simkin, J.; Barth-Wehrenalp, G. J. Inorg. Nucl. Chem. 1962, $24,371-380$.

(36) Yamaguchi, T.; Lindqvist, O. Acta Chem. Scand. 1981, A35, 727-728.

(37) (a) Steffen, W. L.; Palenik, G. J. Acta Cryst. B 1976, 32, 298-300. (b) Qin, J.; Su, N.; Dai, C.; Yang, C.; Liu, D.; Day, M. W.; Wu, B.; Chen, C. Polyhedron 1999, 18, 3461-3464. (c) Roh, S.-G.; Park, Y.-C.; Park, D.-K.; Kim, T.-J.; Jeong, J. H. Polyhedron 2001, 20, 1961-1965, and references therein.

(38) (a) Gorrell, I. B.; Looney, A.; Parkin, G. J. Chem. Soc., Chem. Commun. 1990, 220-222. (b) Looney, A.; Han, R.; Gorrell, I. B.; Cornebise, M.; Yoon, K.; Parkin, G.; Rheingold, A. L. Organometallics 1995, 14, 274-288. (c) Kisko, J. L.; Fillebeen, T.; Hascall, T.; Parkin, G. J. Organomet. Chem. 2000, 596, 22-26. (d) Dias, H. V. R.; Jin, W. Inorg. Chem. 2003, 42, 50345036.

(39) (a) Alsfasser, R.; Powell, A. K.; Trofimenko, S.; Vahrenkamp, H. Chem. Ber. 1993, 126, 685694. (b) Yoon, K.; Parkin, G. J. Am. Chem. Soc. 1991, 113, 8414-8418.

(40) (a) Walker, D. A.; Woodman, T. J.; Schormann, M.; Hughes, D. L.; Bochmann, M. Organometallics 2003, 22, 797-803. (b) Ly, H. V.; Forster, T. D.; Maley, D.; Parvez, M.; Roesler, R. Chem. Commun. 2005, 4468-4470, and references therein.

(41) Fernández, R.; Resa, I.; del Río, D.; Carmona, E.; Gutiérrez-Puebla, E.; Monge, A. Organometallics 2003, 22, 381-383.

(42) Coles, M. P.; Hitchcock, P. B. Eur J Inorg. Chem. 2004, 2662-2672.

(43) Casey, C. P.; O’Connor, J. M. Organometallics 1985, 4, 384-388. 
(44) Mann, B. E. in Comprehensive Organometallic Chemistry Vol. 3. (Eds.: Wilkinson, G.; Stone, F. G. A.; Abel, E. W.), Pergamon, 1982, pp. 89-171, and references therein.

(45) Miele, P.; Foulon, J. D.; Hovnanian, N.; Durand, J.; Cot, L. Eur. J. Solid State Inorg. Chem. 1992, 29, 573-583.

(46) Shibahara, T.; Akashi, H.; Kuroya, H. J. Am. Chem. Soc. 1988, 110, 3313-3314.

(47) See for example: (a) Solanki, N. K.; McInnes, E. J. L.; Collison, D.; Kilner, C. A.; Davies, J. E.; Halcrow, M. A. J. Chem. Soc., Dalton Trans. 2002, 1625-1630. (b) Shimazaki, Y.; Yokoyama, H.; Yamauchi, O. Angew. Chem. Int. Ed. 1999, 38, 2401-2403. (c) Marlin, D. S.; Olmstead, M. M.; Mascharak, P. K. Angew. Chem. Int. Ed. 2001, 40, 4752-4754

(48) (a) Mealli, C.; Arcus, C. S.; Wilkinson, J. L.; Marks, T. J.; Ibers, J. A. J. Am. Chem. Soc. 1976, 98, 711-718. (b) Carrier, S. M.; Ruggiero, C. E.; Houser, R. P.; Tolman, W. B. Inorg. Chem. 1993, 32, 4889-4899. (c) Yoon, K.; Parkin, G. Polyhedron 1995, 14, 811-821. (d) Kiani, S.; Long, J. R.; Stavropoulos, P. Inorg. Chim. Acta. 1997, 263, 357-366.

(49) (a) Kitajima, N.; Koda, T.; Hashimoto, S.; Kitagawa, T.; Moro-oka, Y. J. Am. Chem. Soc. 1991, 113, 5664-5671. (b) Dias, H. V. R.; Jin, W.; Kim, H.-J.; Lu, H.-L. Inorg. Chem. 1996, 35, 2317-2328. (c) Gioia Lobbia, G.; Pettinari, C.; Marchetti, F.; Bovio, B.; Cecchi, P. Polyhedron 1996, 15, 881-890. (d) Gioia Lobbia, G.; Hanna, J. V.; Pellei, M.; Pettinari, C.; Santini, C.; Skelton, B. W.; White, A. H. Dalton Trans. 2004, 951-958. 
Table 1. Experimental data for the X-ray diffraction studies on 3, 8, 9, and 15.

\begin{tabular}{|c|c|c|c|c|}
\hline Formula & $\mathrm{C}_{31} \mathrm{H}_{53} \mathrm{Cl}_{4} \mathrm{~N}_{5} \mathrm{Ti}_{3} \mathrm{Zn}\left(\mathbf{3} \cdot \mathrm{CH}_{2} \mathrm{Cl}_{2}\right)$ & $\mathrm{C}_{45} \mathrm{H}_{68} \mathrm{~N}_{4} \mathrm{SiTi}_{3} \mathrm{Zn}\left(\mathbf{8} \cdot \mathrm{C}_{7} \mathrm{H}_{8}\right)$ & $\mathrm{C}_{36} \mathrm{H}_{65} \mathrm{~N}_{5} \mathrm{Si}_{2} \mathrm{Ti}_{3} \mathrm{Zn}(\mathbf{9})$ & $\mathrm{C}_{66} \mathrm{H}_{100} \mathrm{Cu}_{2} \mathrm{~N}_{8} \mathrm{Ti}_{6}\left(\mathbf{1 5} \cdot \mathrm{C}_{6} \mathrm{D}_{6}\right)$ \\
\hline$M_{\mathrm{r}}$ & 846.65 & 902.19 & 833.18 & 1420.02 \\
\hline$T[\mathrm{~K}]$ & $200(2)$ & $200(2)$ & $200(2)$ & $200(2)$ \\
\hline$\lambda[\AA]$ & 0.71073 & 0.71073 & 0.71073 & 0.71073 \\
\hline crystal system & Monoclinic & Monoclinic & Triclinic & Monoclinic \\
\hline space group & $\mathrm{P} 2_{1} / \mathrm{n}$ & $\mathrm{P} 2_{1} / \mathrm{c}$ & $\mathrm{P}-1$ & $\mathrm{P} 2_{1} / \mathrm{c}$ \\
\hline$a[\AA] ; \alpha\left[^{\circ}\right]$ & $11.595(2)$ & 18.903(2); & $11.645(3) ; 81.76(2)$ & $12.397(2)$ \\
\hline$b[\AA] ; \beta\left[^{\circ}\right]$ & $23.461(2) ; 100.69(1)$ & $11.069(2) ; 101.87(1)$ & $11.906(3) ; 87.73(2)$ & $14.963(3) ; 123.56(1)$ \\
\hline$c[\AA] ; \gamma\left[{ }^{\circ}\right]$ & $14.608(2)$ & $22.510(2)$ & $17.621(4) ; 65.84(2)$ & $22.185(2)$ \\
\hline$V\left[\AA^{3}\right]$ & $3904.7(8)$ & $4609.2(9)$ & $2205.6(9)$ & $3429.3(9)$ \\
\hline $\mathrm{Z}$ & 4 & 4 & 2 & 2 \\
\hline$\rho_{\text {calcd }}\left[\mathrm{g} \mathrm{cm}^{-3}\right]$ & 1.440 & 1.300 & 1.255 & 1.375 \\
\hline$\mu_{\mathrm{MoK} \alpha}\left[\mathrm{mm}^{-1}\right]$ & 1.500 & 1.075 & 1.144 & 1.312 \\
\hline$F(000)$ & 1752 & 1904 & 880 & 1484 \\
\hline crystal size $\left[\mathrm{mm}^{3}\right]$ & $0.3 \times 0.3 \times 0.3$ & $0.3 \times 0.1 \times 0.1$ & $0.33 \times 0.29 \times 0.21$ & $0.41 \times 0.29 \times 0.27$ \\
\hline$\theta$ range & 5.00 to $25.01^{\circ}$ & 5.03 to $25.24^{\circ}$ & 3.05 to $27.50^{\circ}$ & 3.06 to $27.57^{\circ}$ \\
\hline index ranges & -13 to $13,-27$ to $27,-17$ to 17 & -22 to $22,-13$ to $13,-27$ to 27 & -15 to $15,-15$ to $15,-22$ to 22 & -13 to 16,0 to $19,-26$ to 28 \\
\hline Reflections collected & 23861 & 29828 & 18592 & 134861 \\
\hline Unique data & $6817\left[\mathrm{R}_{\mathrm{int}}=0.122\right]$ & $8271\left[\mathrm{R}_{\mathrm{int}}=0.186\right]$ & $9997\left[\mathrm{R}_{\mathrm{int}}=0.042\right]$ & $7908\left[\mathrm{R}_{\mathrm{int}}=0.118\right]$ \\
\hline obsd data $[\mathrm{I}>2 \sigma(\mathrm{I})]$ & 4213 & 3773 & 7640 & 5418 \\
\hline Goodness-of-fit on $\mathrm{F}^{2}$ & 1.042 & 1.092 & 1.065 & 1.087 \\
\hline final $R$ indices $[I>2 \sigma(I)]$ & $\mathrm{R} 1=0.062, \mathrm{wR} 2=0.114$ & $\mathrm{R} 1=0.087, w \mathrm{R} 2=0.172$ & $\mathrm{R} 1=0.047, \mathrm{wR} 2=0.127$ & $\mathrm{R} 1=0.096, \mathrm{wR} 2=0.282$ \\
\hline $\mathrm{R}$ indices (all data) & $\mathrm{R} 1=0.127, \mathrm{wR} 2=0.135$ & $\mathrm{R} 1=0.231, w \mathrm{R} 2=0.231$ & $\mathrm{R} 1=0.067, \mathrm{wR} 2=0.137$ & $\mathrm{R} 1=0.138, \mathrm{wR} 2=0.307$ \\
\hline largest diff. Peak/hole $\left[\mathrm{e} . \AA^{-3}\right]$ & $0.449 /-0.541$ & $0.966 /-1.113$ & $0.763 /-0.674$ & $1.58 /-3.461$ \\
\hline
\end{tabular}

${ }^{\mathrm{a}} R 1=\Sigma|| \mathrm{F}_{0}|-| \mathrm{F}_{\mathrm{c}} \| /\left[\Sigma\left|\mathrm{F}_{0}\right|\right] \quad w R 2=\left\{\left[\Sigma w\left(F_{0}^{2}-F_{c}^{2}\right)^{2}\right] /\left[\Sigma w\left(F_{0}^{2}\right)^{2}\right]\right\}^{1 / 2}$ 